\title{
Cryptic Speciation in Western North America and Eastern Eurasia of the Pathogens Responsible for Laminated Root Rot
}

\author{
I. Leal, ${ }^{\dagger}$ M.-J. Bergeron, N. Feau, C. K. M. Tsui, B. Foord, K. Pellow, R. C. Hamelin, and R. N. Sturrock
}

First, fifth, sixth, and eighth authors: Natural Resources Canada, Canadian Forest Service, Pacific Forestry Centre, 506 West Burnside Road, Victoria, BC V8Z 1M5 Canada; second author: Natural Resources Canada, Canadian Forest Service, Laurentian Forestry Centre, 1055 rue du P.E.P.S., Québec, QC G1V 4C7 Canada; third and seventh authors: Department of Forest and Conservation Sciences, University of British Columbia, 3041-2424 Main Mall, Vancouver, BC V6T 1Z4 Canada; fourth author: Department of Pathology, Sidra Medicine, PO Box 26999, Doha, Qatar, and Department of Pathology and Laboratory Medicine, Weill Cornell Medical College, Doha, Qatar; and seventh author: Institut de Biologie Intégrative et des Systèmes (IBIS), Université Laval/Pavillon Charles-Eugène Marchand, 1030 avenue de la Médecine, Québec, QC G1V 0A6 Canada.

Accepted for publication 21 August 2018.

\begin{abstract}
Coniferiporia sulphurascens is a facultative fungal pathogen that causes laminated root rot (LRR) in commercially important coniferous species worldwide. This fungus spreads primarily by way of vegetative mycelium transferring at points of contact between infected and healthy roots. Successful intervention to control LRR requires a better understanding of the population structure and genetic variability of $C$. sulphurascens. In this study, we investigated the population genetic structure and origin of $C$. sulphurascens populations in western North America and eastern Eurasia collected from multiple coniferous hosts. By analyzing the small and large mitochondrial ribosomal RNA subunit genes combined with six nuclear loci (internal transcribed spacer region, actin, RNA polymerase II largest subunit, RNA polymerase II second-largest subunit, laccase-like multicopper oxidase, and translation elongation factor $1-\alpha$ ), we observed that none of the alleles among the loci were shared between North American (NA) and Eurasian

C. sulphurascens populations. In total, 55 multilocus genotypes (MLGs) were retrieved in $C$. sulphurascens isolates occurring in these two continental regions. Of these, 41 MLGs were observed among 58 isolates collected from widespread locations in British Columbia (Canada) and the northwestern United States, while 14 MLGs were observed among 16 isolates sampled in Siberia and Japan. Our data showed that the levels of genetic differentiation between the NA and Eurasian populations are much greater than the populations from within each continental region; the two continental populations formed clearly divergent phylogenetic clades or lineages since they were separated approximately 7.5 million years ago. Moreover, the Eurasian population could be the source of the NA population. Our study indicates the existence of cryptic diversity in this pathogen species, and strongly suggests that the NA and Eurasian populations represent two lineages, which have progressively diverged from each other in allopatry.
\end{abstract}

The fungus Coniferiporia sulphurascens (Pilát) L. W. Zhou \& Y. C. Dai (formerly known as Phellinus sulphurascens Pilát, syn. P. weirii (Murrill) Gilb. and Phellinidium sulphurascens (Pilát) Y. C. Dai) causes laminated root rot in conifers. It is the most serious pathogen affecting coastal Douglas-fir (Pseudotsuga menziesii (Mirb.) Franco), grand fir (Abies grandis (Dougl. Ex D. Don) Lind1.), and several other conifers in the family Pinaceae in western North America (NA).

This basidiomycete occurs in southern British Columbia, Canada and Washington, Oregon, northern California, western Montana, and northern Idaho in the United States (Buckland et al. 1954; Larsen et al. 1994; Thies and Sturrock 1995), where the coastal and interior varieties of Douglas-fir are naturally distributed (Cook et al. 2013). In these locations, C. sulphurascens has been considered a native species (Holah et al. 1993). The first report in NA of this fungus was in 1929 from specimens collected in Cowichan Lake,

†Corresponding author: I. Leal; E-mail: isabel.leal@canada.ca

Funding: This work was funded through the Genomics Research \& Development Initiative, provided by Natural Resources Canada, Canadian Forest Service with additional funds from Genome Canada and Genome BC.

Isabel Leal and Marie-Josée Bergeron contributed equally to this work.

*The $\boldsymbol{e}$-Xtra logo stands for "electronic extra" and indicates that two supplementary figures and four supplementary tables are published online.

(C) Her Majesty the Queen in Right of Canada, as represented by the Minister of Natural Resources Canada, 2019.
BC, Canada (Mounce et al. 1940). C. sulphurascens has also been isolated from stumps and stems of fallen trees of the genera Abies, Picea, Larix, and Pinus in Eurasia, including in Japan (Aoshima 1953), China (Dai 2010; Dai and Qin 1998), and Siberia (Kotiranta et al. 2005; Parmasto and Parmasto 1979).

Laminated root rot can cause significant growth reduction in forested stands, especially those dominated by Douglas-fir trees (Hansen and Goheen 2000; Thies and Sturrock 1995; Thies and Westlind 2005), increasing the risk of stem breakage and blowdown. Goheen and Hansen (1993) estimated that $C$. sulphurascens causes between 40 and $70 \%$ reduction in wood volume in Oregon and Washington. Although survival, spread, and impact of C. sulphurascens in NA is likely influenced by site conditions, there is no strong evidence yet that any individual site factor or combination of factors reliably predicts the occurrence or intensity of the fungus at a given location (Beale 1992; Kastner et al. 1994; Thies and Sturrock 1995). Impact data and descriptions of damage caused by $C$. sulphurascens in Eurasia are poorly understood, although it has been reported to cause white root and butt rot on Abies spp. in northeastern China. As a result of root rot, stem breakage and uprooting have been observed. This disease has been occasionally reported on species of Picea, Larix, and Pinus, where it causes less damage (Dai 2010; Dai and Qin 1998).

In NA, C. sulphurascens is known to be both a saprophyte and a parasite. Hosts of various ages are susceptible to attack and infection by this fungus. Roots and stumps infected by $C$. sulphurascens can serve as viable inoculum sources for decades (Buckland et al. 1954; Hansen 1979a). The fungus transfers at points of contact between infected and healthy roots and grows along the surface of susceptible 
host roots as ectotrophic mycelium, eventually penetrating the host roots through intact or injured bark. Once inside the root as endotrophic mycelium, the fungus progressively destroys root tissue, depriving the tree of water and nutrients and, thus, weakening its structural support. This vegetative spread results in the development of infection centers. This fungus also disperses basidiospores from brown crustlike sporophores produced episodically on the moist underside of fallen trees. However, there is no evidence that this sexual mode of reproduction plays a significant role in the spread of the fungus (Cook et al. 2013; Hansen 1979b). Whereas sporophores are rare and inconspicuous in NA, especially in interior stands where conditions are generally drier (Cook et al. 2013; Nelson 1971; Thies and Sturrock 1995), they are prevalent during drier seasons in China (Dai and Qin 1998).

Population structure and genetic diversity have been investigated in various root rot pathogens of forest trees such as Armillaria spp. (Baumgartner et al. 2010; Ferguson et al. 2003; Ross-Davis et al. 2012), Heterobasidion spp. (Dalman et al. 2010; Garbelotto et al. 2013; Łakomy et al. 2007; Luchi et al. 2011; Oliva et al. 2011), Neonectria macrodidyma (Menkis and Burokiene 2012), Phytophthora quercina (Cooke et al. 2005), Phellinus torulosus (Campanile et al. 2004), and P. noxius (Akiba et al. 2015; Chung et al. 2017). However, little investigation has been done on the population structure and genetic diversity of $C$. sulphurascens, except for two studies. The first one, a small-scale comparison of NA and Eurasian isolates of C. sulphurascens using both traditional plate pairing and protein and DNA migration patterns in gel electrophoresis, concluded that NA and Eurasian C. sulphurascens isolates represented related but distinct populations (Hansen et al. 1998). In the second population genetic study, conducted by Lim et al. (2008), homokaryons of C. sulphurascens were distinguished from heterokaryons based on the comparative analysis of the internal transcribed spacer (ITS) of nuclear ribosomal RNA gene (rDNA) repeats. Until now, most of the work related to this pathogen focused on deciphering the mechanisms of interaction with the host, through the use of transcriptomics and proteomics approaches. Putative defense- or stress-related genes expressed by Douglas-fir infected by $C$. sulphurascens were identified and characterized (Islam et al. 2008, 2010, 2013); numerous upregulated genes were also characterized from $C$. sulphurascens in infected Douglas-fir (Williams et al. 2014).

Fungal pathogens are often composed of genetically isolated lineages that are not distinguishable on the basis of purely morphological features (Taylor et al. 2006). Detecting the cryptic structure in a pathogen population is essential to understand the biological activities in that pathosystem. A better knowledge of the phylogenetic relationships and genetic diversity of $C$. sulphurascens may help to (i) explain the evolutionary processes and ecological factors that shape pathogen populations and influence their biological adaptation, (ii) detect cryptic species, and (iii) improve disease management by using appropriate pathogen isolates for screening germplasm in breeding programs (Burdon 1993; Burdon and Silk 1997). Here, we used a multilocus genotyping approach (two mitochondrial and six nuclear loci) to investigate the population genetic structure of the fungal pathogen $C$. sulphurascens in two populations: western NA (British Columbia, Canada and the northwestern United States) and eastern Eurasia (Siberia and Japan). We aimed to answer the following questions: (i) are the NA and Eurasian C. sulphurascens populations panmictic or is there evidence of speciation and (ii) what are the evolutionary mechanisms that have driven the occurrence of $C$. sulphurascens population in NA?

\section{MATERIALS AND METHODS}

Sampling and culturing of $\boldsymbol{C}$. sulphurascens and $\boldsymbol{C}$. weirii. In total, $74 C$. sulphurascens isolates from 12 different host species (Abies concolor (Gordon) Lindl. ex Hildebr, A. sachalinensis (F. Schmidt) Mast, Larix gmelinii var. japonica (Maxim. ex Regel) Pilg, L. gmelinii var. principis-ruprechtii (Mayr) Pilg,
L. occidentalis Nutt, Picea engelmannii Parry ex Engelm, $P$. jezoensis (Siebold \& Zucc.) Carrière, P. abies subsp. obovata (Ledeb.) Hultén, Pinus contorta Douglas ex Loudon, P. monticola Douglas ex D. Don, Pseudotsuga menziesii (Mirb.) Franco, and Tsuga heterophylla (Raf.) Sarg.) were collected in NA (British Columbia, Canada, $n=39$ and northwestern United States, $n=19$ ) and Eurasia (Siberia, $n=8$ and Japan, $n=8$ ). The $C$. sulphurascens isolates from British Columbia were collected from four biogeoclimatic zones: Coastal Douglas-fir (CDF), Coastal Western Hemlock (CWH), Interior Cedar-Hemlock (ICH), and Interior Douglas-fir (IDF). These zones are part of a multiscaled, ecosystembased classification system that groups ecologically similar sites based on climate, soils, and vegetation, and which is widely used as a framework for resource management and scientific research in British Columbia (https://www.for.gov.bc.ca/hre/becweb/resources/ classificationreports/provincial/).

Also, six C. weirii (Murrill) L. W. Zhou \& Y. C. Dai (formerly known as Phellinus weirii (Murrill) Gilb., laminated root rot pathogen in cedar in NA) isolates on Thuja plicata Donn ex D. Don were collected from British Columbia, Canada and Idaho, United States (Table 1; Supplementary Table S1). All C. sulphurascens and C. weirii isolates were cultured on $2 \%$ malt extract agar at room temperature (approximately $20^{\circ} \mathrm{C}$ ). In addition, we obtained two herbarium sporophore specimens of Phellinidium cryptocystidiatum Spirin \& Zmitr. (paratype H 7026937 and isotype H 7034465) from Nizhny Novgorod, Russia.

DNA isolation and amplification. DNA was isolated using the Qiagen DNeasy Plant Mini kit (Qiagen Inc., Mississauga, ON, Canada), following the manufacturer's instructions. The DNA was eluted in $100 \mu \mathrm{l}$ of $10 \mathrm{mM}$ Tris- $\mathrm{HCl}, \mathrm{pH} \mathrm{8.0,} \mathrm{and} \mathrm{its} \mathrm{concentration}$ determined by NanoDrop (ThermoFisher Mississauga, ON, Canada). Two mitochondrial ribosomal RNA subunit genes (MS and $M L$ ) and five nuclear coding genes-actin (ACT2), RNA polymerase II largest subunit $(R P B 1)$, RNA polymerase II secondlargest subunit $(R P B 2)$, laccase-like multicopper oxidase $(L A C)$, and translation elongation factor 1- $\alpha(T E F 1)$ - as well as the ITS of rDNA repeats were amplified using the primer pairs described in Table 2 . For $P$. cryptocystidiatum, only $A C T 2, R P B 1$, and $L A C$ genes were amplified.

Polymerase chain reaction (PCR) amplification was performed using $1 \mu \mathrm{l}$ of DNA ( 25 to $50 \mathrm{ng}$ ) as template, in a reaction volume of $25 \mu$ l containing $1 \mathrm{X}$ PCR buffer, $4 \mu \mathrm{g}$ bovine serum albumen (NEB, Whitby, ON, Canada), $1.6 \mathrm{mM} \mathrm{MgCl} 2,200 \mu \mathrm{M}$ each dNTP (NEB, USA), $1 \mu \mathrm{M}$ each primer (Integrated DNATechnologies, Coralville, IA), and $1 \mathrm{U}$ of Platinum Taq DNA polymerase (Invitrogen, Life Technologies, Carlsbad, CA, USA). Amplification was carried out in a Whatman Biometra T-Gradient thermocycler (Biometra $\mathrm{GmbH}$, Goettingen, Germany) with an initial denaturation step of $5 \mathrm{~min}$ at $94^{\circ} \mathrm{C}$; followed by 35 cycles of denaturation, primer annealing; and elongation at $72^{\circ} \mathrm{C}$ for $1 \mathrm{~min}$ (Table 2); with a final extension step at $72^{\circ} \mathrm{C}$ for $10 \mathrm{~min}$.

Multilocus genotyping, cloning, and sequencing. Multilocus genotypes (MLGs) of all $C$. sulphurascens isolates were obtained by both single-strand conformation polymorphism (SSCP) analyses and sequencing (Hamelin et al. 2005). Initial screening of polymorphism for $M S, M L, A C T 2, R P B 1, R P B 2$, and $T E F 1$ was first carried out using the SSCP method. Specific running conditions, including temperature, electrical power, and migration time, were empirically determined as follows: $T E F 1: 4^{\circ} \mathrm{C}, 2 \mathrm{~W}$, $18 \mathrm{~h}$; and $A C T 2, R P B 1, R P B 2, M S$, and $M L: 20^{\circ} \mathrm{C}, 2 \mathrm{~W}, 18 \mathrm{~h}$. The alleles were then scored visually according to their migration profiles and representative samples were selected for sequencing. Those that showed unique allele were sequenced twice, in both directions, to confirm their distinctive character. The nuclear ITS region was genotyped directly by sequencing, without using the SSCP method.

Cloning was performed to determine the exact nucleotide sequence of haplotypes for heterozygous samples displaying more 
than one polymorphic site within their sequences. PCR products used in cloning experiments were purified using the QIAquick PCR purification kit (Qiagen Inc.) and ligated into the pDrive cloning vector provided in the Qiagen PCR cloningplus kit (Qiagen Inc.), according to the manufacturer's instructions, with the following modification: $0.7 \mu$ l of ligation master mix was used during the ligation reaction. The transformation was conducted using $15 \mu \mathrm{l}$ of competent cells which were combined with $80 \mu \mathrm{l}$ of Super Optimal broth with Catabolite repression (SOC) media, then incubated for $30 \mathrm{~min}$ at $37^{\circ} \mathrm{C}$ with shaking. Bacterial culture aliquots were plated onto selective media containing ampicillin at $100 \mu \mathrm{g} / \mathrm{ml}$, 5-bromo4-chloro-3-indolyl- $\beta$-D-galactoside (X-gal) at $80 \mu \mathrm{g} / \mathrm{ml}$, and $50 \mu \mathrm{M}$ isopropyl- $\beta$-D-thiogalactopyranoside (IPTG). Plates were then incubated overnight at $37^{\circ} \mathrm{C}$. Individual white colonies were isolated and grown overnight in Luria-Bertani broth containing ampicillin at $100 \mu \mathrm{g} / \mathrm{ml}$. Plasmids were harvested from the cultures using a phenol/chloroform extraction method (Sambrook et al. 1989).

All sequencing was carried out at Genome sequencing and genotyping platform of the CHUL Research Center, Québec,

TABLE 1. Coniferiporia sulphurascens and C. weirii isolates used in this study ${ }^{\mathrm{w}}$

\begin{tabular}{|c|c|c|c|c|c|c|c|c|c|c|c|c|}
\hline \multirow[b]{2}{*}{ Isolate } & \multirow[b]{2}{*}{ Host } & \multirow[b]{2}{*}{ Collection site } & \multirow[b]{2}{*}{ Zone $^{y}$} & \multirow[b]{2}{*}{$\mathrm{MLG}^{\mathrm{z}}$} & \multicolumn{8}{|c|}{ Allele types ${ }^{x}$} \\
\hline & & & & & ITS & $M S$ & $M L$ & $R P B 1$ & $A C T 2$ & TEF1 & $R P B 2$ & $L A C$ \\
\hline \multicolumn{13}{|l|}{ C. sulphurascens } \\
\hline 505 & $\begin{array}{l}\text { Pseudotsuga } \\
\text { menziesii }\end{array}$ & Canada, BC, French Ck. & $\mathrm{CDF}$ & 37 & 2 & a & $\mathrm{a}$ & $\mathrm{ac}$ & aa & aa & aa & aa \\
\hline 606 & P. menziesii & Canada, BC, Quamichan Bay & $\mathrm{CDF}$ & 6 & 1 & a & $\mathrm{a}$ & $\mathrm{cc}$ & $\mathrm{aa}$ & $\mathrm{ac}$ & aa & aj \\
\hline 631 & P. menziesii & $\begin{array}{l}\text { Canada, BC, Little Qualicum } \\
\text { River Park }\end{array}$ & $\mathrm{CDF}$ & 27 & $1 \& 2$ & a & $\mathrm{c}$ & aa & aa & aa & ac & $\mathrm{bb}$ \\
\hline 612 & P. menziesii & Canada, BC, Victoria & $\mathrm{CDF}$ & 14 & $1 \& 2$ & a & $\mathrm{a}$ & aa & aa & aa & $\mathrm{ac}$ & ak \\
\hline 578 & Pinus contorta & Canada, BC, Goldstream Park & $\mathrm{CDF}$ & 36 & 2 & a & $\mathrm{a}$ & $a b$ & aa & aa & ac & aa \\
\hline 577 & P. menziesii & Canada, BC, Saanich & $\mathrm{CDF}$ & 31 & 2 & a & $\mathrm{a}$ & aa & aa & aa & aa & $\mathrm{bb}$ \\
\hline 610 & P. menziesii & Canada, BC, Victoria & $\mathrm{CDF}$ & 3 & 1 & a & $\mathrm{a}$ & aa & aa & aa & $\mathrm{ac}$ & aa \\
\hline 605 & P. menziesii & Canada, BC, Metchosin & $\mathrm{CDF}$ & 21 & $1 \& 2$ & a & $\mathrm{a}$ & $\mathrm{ac}$ & aa & aa & aa & $\mathrm{ab}$ \\
\hline 633 & P. menziesii & Canada, BC, South Surrey & $\mathrm{CDF}$ & 11 & $1 \& 2$ & $\mathrm{a}$ & $\mathrm{a}$ & aa & aa & aa & $\mathrm{ac}$ & $a b$ \\
\hline 602 & P. menziesii & Canada, BC, Gold River & CWH & 2 & 1 & a & $\mathrm{a}$ & aa & aa & aa & aa & $a b$ \\
\hline 584 & P. menziesii & Canada, BC, Port McNeil & CWH & 32 & 2 & a & $\mathrm{a}$ & aa & aa & aa & $\mathrm{ac}$ & $a b$ \\
\hline 503 & Pinus monticola & Canada, BC, Cowichan Lake & CWH & 22 & $1 \& 2$ & a & a & $\mathrm{ah}$ & aa & aa & $\mathrm{ac}$ & $a b$ \\
\hline 581 & P. menziesii & Canada, BC, Cowichan Lake & CWH & 2 & 1 & a & $\mathrm{a}$ & aa & aa & aa & aa & $a b$ \\
\hline 583 & P. menziesii & Canada, BC, Shawnigan Lake & CWH & 19 & $1 \& 2$ & a & $\mathrm{a}$ & ac & aa & ac & aa & $a b$ \\
\hline 527 & P. menziesii & $\begin{array}{l}\text { Canada, BC, Harrison Hot } \\
\text { Springs }\end{array}$ & $\mathrm{CWH}$ & 15 & $1 \& 2$ & a & $\mathrm{a}$ & aa & aa & aa & $\mathrm{ac}$ & $\mathrm{bb}$ \\
\hline 514 & P. menziesii & Canada, BC, Chilliwack & CWH & 4 & 1 & a & $\mathrm{a}$ & aa & aa & $\mathrm{ac}$ & bc & bc \\
\hline 509 & $\begin{array}{l}\text { Tsuga } \\
\text { heterophylla }\end{array}$ & Canada, BC, Chilliwack & CWH & 13 & $1 \& 2$ & a & $\mathrm{a}$ & aa & aa & aa & dd & aa \\
\hline 591 & P. menziesii & Canada, BC, Sechelt & CWH & 8 & $1 \& 2$ & a & a & aa & aa & aa & aa & aa \\
\hline 601 & T. heterophylla & Canada, BC, Texada Island & CWH & 20 & $1 \& 2$ & a & $\mathrm{a}$ & $\mathrm{ac}$ & am & aa & aa & $a b$ \\
\hline 548 & P. menziesii & Canada, BC, Fauquier & $\mathrm{ICH}$ & 30 & 2 & a & $\mathrm{a}$ & aa & $\mathrm{ab}$ & aa & aa & $\mathrm{jj}$ \\
\hline 547 & P. menziesii & Canada, BC, Fauquier & $\mathrm{ICH}$ & 30 & 2 & a & $\mathrm{a}$ & aa & $a b$ & aa & aa & $\mathrm{jj}$ \\
\hline 546 & P. menziesii & Canada, BC, Slocan & $\mathrm{ICH}$ & 29 & 2 & a & $\mathrm{a}$ & aa & $a b$ & aa & aa & aj \\
\hline 544 & P. menziesii & Canada, BC, Nelson & $\mathrm{ICH}$ & 5 & 1 & a & $\mathrm{a}$ & aa & $a b$ & aa & ad & aa \\
\hline 565 & P. menziesii & Canada, BC, New Denver & $\mathrm{ICH}$ & 8 & $1 \& 2$ & a & $\mathrm{a}$ & aa & aa & aa & aa & aa \\
\hline 566 & P. menziesii & Canada, BC, Nakusp & $\mathrm{ICH}$ & 26 & $1 \& 2$ & a & $\mathrm{a}$ & aa & $a b$ & aa & aa & $\mathrm{bb}$ \\
\hline 568 & P. menziesii & Canada, BC, Lumby, Sugar Lk & $\mathrm{ICH}$ & 28 & 2 & a & $\mathrm{a}$ & aa & aa & aa & aa & aa \\
\hline 623 & Larix occidentalis & Canada, BC, Enderby & $\mathrm{ICH}$ & 9 & $1 \& 2$ & a & $\mathrm{a}$ & aa & aa & aa & an & $\mathrm{bb}$ \\
\hline 635 & P. menziesii & Canada, BC, Seymour Arm & $\mathrm{ICH}$ & 18 & $1 \& 2$ & a & $\mathrm{a}$ & $a b$ & aa & aa & ac & $\mathrm{bb}$ \\
\hline 538 & P. contorta & Canada, BC, Boston Bar & IDF & 2 & 1 & a & $\mathrm{a}$ & aa & aa & aa & aa & $a b$ \\
\hline 524 & P. menziesii & Canada, BC, Boston Bar & IDF & 17 & $1 \& 2$ & a & $\mathrm{a}$ & aa & aa & $a b$ & aa & $\mathrm{bb}$ \\
\hline 569 & P. menziesii & Canada, BC, Salmon Arm & IDF & 8 & $1 \& 2$ & a & $\mathrm{a}$ & aa & aa & aa & aa & aa \\
\hline 603 & P. menziesii & Canada, BC, Skimikan & IDF & 1 & 1 & a & $\mathrm{a}$ & aa & aa & aa & aa & aa \\
\hline 553 & P. menziesii & Canada, BC, Lumby, Mabel Lk & IDF & 8 & $1 \& 2$ & a & $\mathrm{a}$ & aa & aa & aa & aa & aa \\
\hline 574 & Picea engelmannii & Canada, BC, Merritt & IDF & 21 & $1 \& 2$ & a & $\mathrm{a}$ & $\mathrm{ac}$ & aa & aa & aa & $a b$ \\
\hline 541 & P. menziesii & Canada, BC, Princeton & IDF & 12 & $1 \& 2$ & $\mathrm{a}$ & $\mathrm{a}$ & aa & aa & aa & aa & $a b$ \\
\hline 536 & P. menziesii & Canada, BC, Squamish & IDF & 10 & $1 \& 2$ & $\mathrm{a}$ & $\mathrm{a}$ & aa & aa & aa & ae & aa \\
\hline 533 & P. menziesii & Canada, BC, Squamish & IDF & 9 & $1 \& 2$ & a & $\mathrm{a}$ & aa & aa & aa & an & $\mathrm{bb}$ \\
\hline 556 & P. menziesii & Canada, BC, Kamloops, BC. & IDF & 16 & $1 \& 2$ & a & $\mathrm{a}$ & aa & aa & aa & aa & $\mathrm{bb}$ \\
\hline Ps5-wf & Abies concolor & $\begin{array}{l}\text { United States, CA, Humboldt } \\
\text { County }\end{array}$ & $\ldots$ & 41 & 1 & a & $\mathrm{a}$ & ac & aa & aa & $\mathrm{cn}$ & $\mathrm{bb}$ \\
\hline Ps6-wf & P. menziesii & $\begin{array}{l}\text { United States, CA, Humboldt } \\
\text { County }\end{array}$ & $\ldots$ & 41 & 1 & a & $\mathrm{a}$ & $\mathrm{ac}$ & aa & aa & $\mathrm{cn}$ & $\mathrm{bb}$ \\
\hline Ps7-wf & A. concolor & $\begin{array}{l}\text { United States, CA, Humboldt } \\
\text { County }\end{array}$ & $\ldots$ & 41 & 1 & a & $\mathrm{a}$ & $\mathrm{ac}$ & aa & aa & $\mathrm{cn}$ & $\mathrm{bb}$ \\
\hline 104 & P. menziesii & United States, ID, Coeur d'Alene & $\ldots$ & 35 & 2 & a & $\mathrm{a}$ & aa & $\mathrm{mn}$ & aa & ao & $\mathrm{bb}$ \\
\hline FP-134842-T & P. menziesii & United States, ID, Coeur d'Alene & $\ldots$ & 39 & 2 & a & $\mathrm{a}$ & aa & ai & ac & ac & $\mathrm{bb}$ \\
\hline FP-134847-T & P. menziesii & United States, ID, Coeur d'Alene & $\ldots$ & 34 & 2 & a & $\mathrm{a}$ & aa & aa & aa & aa & $a b$ \\
\hline FP-91601-T & P. menziesii & United States, WA, LaGrande & $\cdots$ & 25 & $1 \& 2$ & a & $\mathrm{a}$ & $\mathrm{ac}$ & aa & aa & $\mathrm{ac}$ & aa \\
\hline & & & & & & & & & & & ned & lext pa \\
\hline
\end{tabular}

w The origin of each isolate is specified in the National Center for Biotechnology Information under each corresponding accession number.

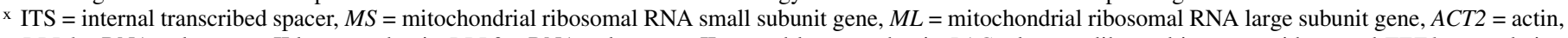
$R P B 1=$ RNA polymerase II largest subunit, $R P B 2=$ RNA polymerase II second-largest subunit, $L A C=$ laccase-like multicopper oxidase, and $T E F 1=$ translation elongation factor $1-\alpha$.

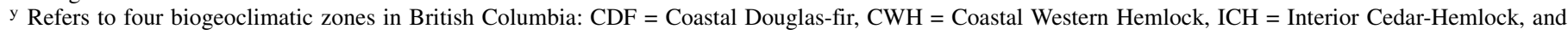
$\mathrm{IDF}=$ Interior Douglas-fir.

z Multilocus genotypes. 
Canada. Sequence data were edited using Sequencher software 4.8 (Gene Codes Corporation, Ann Arbor, MI, USA) or Geneious 6.1.2 (Biomatters Ltd., Auckland, New Zealand) (https://www.geneious. $\mathrm{com} /$ ), and deposited into the GenBank database (Table 2).

Genetic structure analysis. To analyze and visualize the genetic differences among the 74 isolates of C. sulphurascens from NA and Eurasia as a function of geographical origin (including the four biogeoclimatic zones for the British Columbia isolates) and hosts, principal coordinate analysis (PCoA) implemented in Exceladd-in software GenAlEx 6.502 (Peakall and Smouse 2006, 2012) was performed. Molecular variance was also estimated using GenAlEx. Dnasp 5.10.01 (Librado and Rozas 2009) was used to summarize polymorphism information and genetic differentiation among populations.

Population structure was assessed by using STRUCTURE 2.3.4, a Bayesian clustering algorithm, which assigns individuals into genetic clusters and quantifies levels of admixture (https://web.stanford.edu/group/pritchardlab/structure.html) (Hubisz et al. 2009). This was carried out by analyzing the five nuclear genes, including 116 segregating sites. The analysis was run for $K$ clusters ranging from 1 to 10 , and each $K$ was replicated 10 times with a burn-in length of 100,000 generations followed by an additional 500,000 generations to verify the likelihood values of each value of $K$. STRUCTURE was set to run with mixed ancestry (admixture) and an independent allele frequencies model. The spatial sampling location of each population was either included or not as input prior. STRUCTURE HARVESTER was used to estimate the optimal $K$ value with the highest likelihood (http://taylorO.biology.ucla.edu/struct_harvest) (Earl and vonHoldt 2012), based on the results generated by the program STRUCTURE.

Index of association. We used a multilocus variance test to evaluate the overall association among nuclear loci and determine the degree of clonality in NA and Eurasian populations of

TABLE 1. (Continued from previous page)

\begin{tabular}{|c|c|c|c|c|c|c|c|c|c|c|c|c|}
\hline \multirow[b]{2}{*}{ Isolate } & \multirow[b]{2}{*}{ Host } & \multirow[b]{2}{*}{ Collection site } & \multirow[b]{2}{*}{ Zone $^{y}$} & \multirow[b]{2}{*}{$\mathrm{MLG}^{\mathrm{z}}$} & \multicolumn{8}{|c|}{ Allele types ${ }^{x}$} \\
\hline & & & & & ITS & $M S$ & $M L$ & $R P B 1$ & $A C T 2$ & $T E F 1$ & $R P B 2$ & $L A C$ \\
\hline GR-762-4 & P. menziesii & $\begin{array}{l}\text { United States, OR, Grande } \\
\text { Rhonde }\end{array}$ & $\cdots$ & 24 & $1 \& 2$ & a & a & $\mathrm{ac}$ & aa & aa & ac & $\mathrm{ab}$ \\
\hline Mat-9-2 & P. menziesii & United States, WA, Matlock & $\ldots$ & 38 & 2 & a & a & $\mathrm{ac}$ & aa & aa & aa & $a b$ \\
\hline PW-DF-A3-10 & P. menziesii & $\begin{array}{l}\text { United States, OR, Lobster } \\
\text { Valley }\end{array}$ & $\ldots$ & 31 & 2 & $\mathrm{a}$ & $\mathrm{a}$ & aa & aa & aa & aa & $\mathrm{bb}$ \\
\hline $\mathrm{T}-124$ & P. menziesii & United States, WA, Quilcine & $\ldots$ & 23 & $1 \& 2$ & a & a & aa & $a b$ & aa & $\mathrm{cc}$ & aa \\
\hline JH-50-WR-df & P. menziesii & United States, WA, Carson & $\ldots$ & 40 & $1 \& 12$ & a & a & aa & $a b$ & aa & aa & $a b$ \\
\hline 71101 & P. menziesii & $\begin{array}{l}\text { United States MT, Lolo Nat'l } \\
\text { Forest }\end{array}$ & $\ldots$ & 2 & 1 & a & $\mathrm{a}$ & aa & aa & aa & aa & $a b$ \\
\hline 71102 & P. menziesii & $\begin{array}{l}\text { United States MT, Lolo Nat'l } \\
\text { Forest }\end{array}$ & $\ldots$ & 2 & 1 & a & $\mathrm{a}$ & aa & aa & aa & aa & $\mathrm{ab}$ \\
\hline 71103 & P. menziesii & $\begin{array}{l}\text { United States MT, Lolo Nat'1 } \\
\text { Forest }\end{array}$ & $\ldots$ & 2 & 1 & $\mathrm{a}$ & $\mathrm{a}$ & aa & aa & aa & aa & $\mathrm{ab}$ \\
\hline 71104 & P. menziesii & $\begin{array}{l}\text { United States MT, Lolo Nat'l } \\
\text { Forest }\end{array}$ & $\ldots$ & 2 & 1 & a & $\mathrm{a}$ & aa & aa & aa & aa & $a b$ \\
\hline 80501 & P. menziesii & $\begin{array}{l}\text { United States, ID, Kootenai Nat'1 } \\
\text { Forest }\end{array}$ & $\ldots$ & 33 & 2 & a & $\mathrm{a}$ & aa & aa & aa & $\mathrm{ad}$ & $a b$ \\
\hline 80502 & P. menziesii & $\begin{array}{l}\text { United States, ID, Kootenai Nat'1 } \\
\text { Forest }\end{array}$ & $\ldots$ & 33 & 2 & $\mathrm{a}$ & $\mathrm{a}$ & aa & aa & aa & $\mathrm{ad}$ & $\mathrm{ab}$ \\
\hline WFIStmp2 & A. concolor & $\begin{array}{l}\text { United States, CA, Klamath Nat'1 } \\
\text { Forest }\end{array}$ & $\ldots$ & 7 & 1 & a & $\mathrm{a}$ & aa & aa & aa & aa & ak \\
\hline 3305 & Larix sp. & Siberia, Primorsk, Maise & $\ldots$ & 54 & $3 \& 4$ & $\mathrm{~b}$ & b & dd & $\mathrm{cd}$ & $\mathrm{ff}$ & 11 & dd \\
\hline 3228 & Larix sp. & Siberia, Primorsk, Maise & $\ldots$ & 55 & 5 & $\mathrm{~b}$ & $\mathrm{~b}$ & ee & ef & hh & 11 & ee \\
\hline 8106 & $\begin{array}{l}\text { Picea abies subsp. } \\
\text { obovata }\end{array}$ & Siberia, Buguldeika, Irkusk & $\cdots$ & 51 & $14 \& 15$ & $\mathrm{~b}$ & $\mathrm{~b}$ & $\mathrm{gl}$ & df & ee & ls & eg \\
\hline 8110 & $\begin{array}{l}\text { P. abies subsp. } \\
\text { obovata }\end{array}$ & Siberia, Buguldeika, Irkusk & $\ldots$ & 49 & $13 \& 20$ & $\mathrm{~b}$ & $\mathrm{~b}$ & $\mathrm{kk}$ & $\mathrm{gk}$ & fh & tu & ee \\
\hline 8111 & $\begin{array}{l}\text { P. abies subsp. } \\
\text { obovata }\end{array}$ & Siberia, Buguldeika, Irkusk & $\cdots$ & 53 & $16 \& 17$ & $\mathrm{c}$ & $\mathrm{b}$ & $\operatorname{lm}$ & dd & fh & lq & ef \\
\hline 8116 & $\begin{array}{l}\text { P. abies subsp. } \\
\text { obovata }\end{array}$ & Siberia, Buguldeika, Irkusk & $\cdots$ & 52 & $16 \& 18$ & $\mathrm{~b}$ & $\mathrm{~b}$ & $\mathrm{ij}$ & go & ef & lr & $\mathrm{fm}$ \\
\hline 8117 & $\begin{array}{l}\text { P. abies subsp. } \\
\text { obovata }\end{array}$ & Siberia, Buguldeika, Irkusk & $\cdots$ & 52 & $16 \& 18$ & $\mathrm{~b}$ & $\mathrm{~b}$ & $\mathrm{ij}$ & go & ef & $\operatorname{lr}$ & fm \\
\hline 8122 & $\begin{array}{l}\text { P. abies subsp. } \\
\text { obovata }\end{array}$ & Siberia, Vydrino, Irkusk & $\cdots$ & 47 & $7 \& 20$ & $\mathrm{~b}$ & $\mathrm{~b}$ & $\mathrm{jk}$ & $\mathrm{pq}$ & eh & lv & en \\
\hline FH-1127 & P. jezoensis & Japan, Hokkaido, Kamikawa & $\ldots$ & 46 & $4 \& 8$ & $\mathrm{~b}$ & $\mathrm{~b}$ & gg & fg & de & 11 & ee \\
\hline FH-1230 & A. sachalinensis & Japan, Hokkaido, Kamikawa & $\ldots$ & 42 & $10 \& 19$ & $\mathrm{~b}$ & b & gg & $\mathrm{dj}$ & gg & fl & eg \\
\hline FH-1241 & P. jezoensis & Japan, Hokkaido, Oketo & $\ldots$ & 43 & $4 \& 6$ & $\mathrm{~b}$ & $\mathrm{~b}$ & fg & hh & ee & lp & ee \\
\hline FH-1246 & $\begin{array}{l}\text { L. gmelinii var. } \\
\text { japonica }\end{array}$ & Japan, Hokkaido, Kamikawa & $\cdots$ & 50 & $9 \& 10$ & $\mathrm{~b}$ & $\mathrm{~b}$ & $\mathrm{ff}$ & gj & ef & 11 & hi \\
\hline FH-1301 & P. jezoensis & Japan, Hokkaido, Kamikawa & $\ldots$ & 44 & $4 \& 7$ & $\mathrm{~b}$ & b & gn & $\mathrm{kl}$ & ef & $\operatorname{lm}$ & el \\
\hline FH-1313 & P. jezoensis & Japan, Hokkaido, Kamikawa & $\ldots$ & 45 & $4 \& 7$ & $\mathrm{~b}$ & $\mathrm{~b}$ & fg & $\mathrm{jk}$ & ef & $\mathrm{gl}$ & el \\
\hline FH-1506 & A. sachalinensis & Japan, Hokkaido, Oketo & $\ldots$ & 48 & $8 \& 11$ & $\mathrm{~b}$ & b & gg & hh & fg & 11 & eg \\
\hline FH-1507 & $\begin{array}{l}\text { L. gmelinii var. } \\
\text { principis- } \\
\text { ruprechtii }\end{array}$ & Japan, Hokkaido, Oketo & $\ldots$ & 48 & $8 \& 11$ & $\mathrm{~b}$ & $\mathrm{~b}$ & gg & hh & fg & 11 & eg \\
\hline \multicolumn{13}{|l|}{ C. weirii } \\
\hline 504 & Thuja plicata & Canada, BC & $\ldots$ & $\ldots$ & Pw-a & Pw-a & Pw-a & Pw-aa & Pw-ab & Pw-ab & Pw-aa & Pw-aa \\
\hline 545 & T. plicata & Canada, BC & $\ldots$ & $\ldots$ & $\mathrm{Pw}-\mathrm{a}$ & $\mathrm{Pw}-\mathrm{a}$ & Pw-a & Pw-aa & Pw-ab & Pw-aa & Pw-aa & Pw-aa \\
\hline 549 & T. plicata & Canada, BC & $\ldots$ & $\ldots$ & Pw-a & Pw-a & Pw-a & Pw-aa & Pw-ad & Pw-aa & Pw-aa & Pw-aa \\
\hline 586 & T. plicata & Canada, BC & $\ldots$ & $\ldots$ & $\mathrm{Pw}-\mathrm{a}$ & $\mathrm{Pw}-\mathrm{a}$ & $\mathrm{Pw}-\mathrm{a}$ & Pw-bb & Pw-ad & Pw-aa & Pw-aa & Pw-aa \\
\hline 30910 & T. plicata & United States, ID & $\ldots$ & $\ldots$ & $\mathrm{Pw}-\mathrm{a}$ & Pw-a & Pw-a & Pw-aa & Pw-aa & Pw-ab & Pw-aa & Pw-aa \\
\hline 30927 & T. plicata & United States, ID & $\ldots$ & $\ldots$ & Pw-a & Pw-a & Pw-a & Pw-ab & Pw-ac & Pw-bb & Pw-aa & Pw-aa \\
\hline
\end{tabular}


C. sulphurascens (Agapow and Burt 2001; Smith et al. 1993). The index of association $\left(\mathrm{I}_{\mathrm{A}}\right)$ and the normalized value $\bar{r}_{\mathrm{d}}$ (used to remove sample size dependency) were calculated and compared with the distribution obtained if the population is under the hypothesis of complete panmixia by using the poppr R package (Kamvar et al. 2014).

Single- and concatenated-gene phylogeny. Determination of the optimal substitution model for each of the six nuclear gene was conducted with IQ-TREE (Trifinopoulos et al. 2016). Phylogenetic analyses were conducted on each of the six nuclear genes as well as the combined dataset of these genes, which encompasses, in total, four partitions: one partition for $A C T 2, R P B 1$, and TEF1 $(\mathrm{SYM}+\mathrm{I}+\mathrm{G})$; one partition for $R P B 2(\mathrm{~K} 80+\mathrm{I}+\mathrm{G})$; one partition for $L A C$ (TPM2+G); as well as one partition for the ITS1 and ITS2 (JC), for a total of 3,214 bp. The $n S S U$ and $n L S U$ regions neighboring each ITS as well as the $5.8 \mathrm{~S}$ were excluded because no polymorphism was observed within these regions.

Bayesian analyses were conducted in MrBayes 3.2.2 (Ronquist and Huelsenbeck 2003). Two simultaneous and independent searches of the posterior probability distribution, starting from different randomly chosen trees, were run with one cold and three incrementally heated chains for 3 million generations, sampled every 100 generations. The first 5,000 burn-in samples were discarded and the remaining samples of trees were pooled to construct a majority rule consensus tree which was visualized in FigTree 1.4.0 (http://tree.bio.ed.ac.uk/software/figtree/).

Phylogenetic reconstruction based on maximum likelihood (ML) was performed with the RAxML 8.2 using the GTR model with a GAMMA model of rate heterogeneity among sites and a proportion of invariable sites, depending on the model predicted by IQ-TREE (Stamatakis 2014). Bootstrap proportions were determined by 1,000 iterations and visualized on the shortest ML tree with FigTree. The ML tree file was deposited in Treebase (TB2:S21895; https:// treebase.org/treebase-web/home.html).

Divergence time estimation. ML trees among 25 fungal taxa, including three species of Ascomycota and Basidiomycota of the Hymenochaetales $(n=8)$, Polyporales $(n=2)$, Russulales $(n=2)$, Boletales $(n=2)$, Agaricales $(n=5)$, Tremellales $(n=2)$, and Ustilaginales $(n=1)$ orders and six representatives of C. sulphurascens, were inferred by fast and effective stochastic algorithm implemented in FastTree (Price et al. 2010). BEAST 1.8.3 software (Drummond and Rambaut 2007) was used to estimate the divergence time between the NA and Eurasian populations of $C$. sulphurascens based on conservative dates (using the Ascomycota-Basidiomycota divergence time of 582 to 843 million years ago [Mya]) that encompass values obtained by Kasuga et al. (2002) and Berbee and Taylor (2010). We added two internal calibration points corresponding to the fossil ancestors of the orders Boletales and Agaricales (113 Mya and a standard deviation of 1.0) (Riess et al. 2016) and the Cryptococcus gatti-C. neoformans split (49 Mya) (Ngamskulrungroj et al. 2009). Bayesian interference branch support was also inferred by posterior probabilities. The four nuclear gene datasets ( $A C T 2, R P B 1, R P B 2$, and $T E F 1 ; L A C$ was not included due to missing data in other basidiomycetes) were analyzed in two different partitions based on substitution model predictions from IQ-TREE: $A C T 2, R P B 1$, and TEF1 with $\mathrm{SYM}+\mathrm{I}+\mathrm{G}$ and $R P B 2$ with $\mathrm{K} 80+\mathrm{I}+\mathrm{G}$. The uncorrelated lognormal relaxed clock model was used in molecular clock analysis. Tree prior was set to Yule speciation with birth-death process and the final *.xml file was then executed in BEAST. Three independent runs of 40 million generations each were performed. Convergence of all runs, parameter effective sample sizes, burn-in determination, and $95 \%$ highest posterior densities (HPDs) of parameters were evaluated in Tracer 1.6 (http://tree.bio.ed.ac.uk/software/tracer/). Consolidation of run results was performed in LogCombiner 1.8.3 (Drummond and Rambaut 2007) after removing the 10,000 burnins of each independent run, and the resulting tree was visualized with FigTree.

\section{RESULTS}

Genetic diversity and structure of Coniferiporia sulphurascens populations in NA and Eurasia. The Eurasian population showed the greatest genotypic, haplotypic, and genetic diversity in comparison with the NA population. In all, 41 distinct MLGs were observed among 58 isolates sampled at widespread locations in British Columbia and northwestern United States. Of those 41 MLGs, 5 (MLG2, MLG8, MLG21, MLG28, and MLG31) were found at more than one location (Fig. 1A; Table 1). The

TABLE 2. Gene description, primer sequence, polymerase chain reaction parameters, sequence length, and National Center for Biotechnology Information (NCBI) accession numbers

\begin{tabular}{|c|c|c|c|c|c|c|c|}
\hline \multirow[b]{2}{*}{$\begin{array}{l}\text { Gene description } \\
\text { (abbreviation) }\end{array}$} & \multirow{2}{*}{\multicolumn{2}{|c|}{ Primer pairs (reference) and sequences ${ }^{\mathrm{y}}$}} & \multirow[b]{2}{*}{ Annealing } & \multirow[b]{2}{*}{$\mathrm{Sp}^{\mathrm{z}}$} & \multicolumn{2}{|c|}{ Sequence length (bp) } & \multirow[b]{2}{*}{ NCBI } \\
\hline & & & & & Total & $\begin{array}{l}\text { Exon } \\
\text { (intron) }\end{array}$ & \\
\hline \multirow{2}{*}{$\begin{array}{l}\text { Mitochondrial small } \\
\text { ribosomal RNA } \\
\text { (rRNA) subunit }(M S)\end{array}$} & MS1 (a) & CAGCAGTCAAGAATATTAGTCAATG & $55^{\circ} \mathrm{C}, 30 \mathrm{~s}$ & Cs & 760 & $\cdots$ & KX881847-KX881849 \\
\hline & MS2 (a) & GCGGATTATCGAATTAAATAAC & $\cdots$ & $\mathrm{Cw}$ & 772 & $\cdots$ & KX830866 \\
\hline \multirow{2}{*}{$\begin{array}{l}\text { Mitochondrial large } \\
\text { rRNA subunit }(M L)\end{array}$} & ML5 (a) & CTCGGCAAATTATCCTCATAAG & $55^{\circ} \mathrm{C}, 30 \mathrm{~s}$ & Cs & $706-707$ & $\ldots$ & KX881844-KX881846 \\
\hline & ML6 (a) & CAGTAGAAGCTGCATAGGGTC & $\ldots$ & $\mathrm{Cw}$ & 770 & $\ldots$ & KX830865 \\
\hline \multirow{2}{*}{$\begin{array}{l}\text { Nuclear 5.8S rRNA } \\
\text { gene and flanking } \\
\text { internal transcribed } \\
\text { spacers (ITS) }\end{array}$} & ITS5 (a) & GGAAGTAAAAGTCGTAACAAGG & $50^{\circ} \mathrm{C}, 60 \mathrm{~s}$ & Cs & $630-638$ & $\ldots$ & KX881835-KX881843 \\
\hline & ITS4 (a) & TCCTCCGCTTATTGATATGC & $\cdots$ & $\mathrm{Cw}$ & 636 & $\cdots$ & AY829342 \\
\hline \multirow{2}{*}{$\begin{array}{l}\text { Largest subunit of RNA } \\
\text { polymerase I }(R P B I)\end{array}$} & pwrpb1-32f (e) & AGGATACACTTTGTGGTGTCCG & $55^{\circ} \mathrm{C}, 30 \mathrm{~s}$ & Cs & 571 & 571 & KX881850-KX881863 \\
\hline & pwrpb1-643rc (e) & AGTGATCACGCGCCAAGTT & $\ldots$ & $\mathrm{Cw}$ & 571 & 571 & KX830867-KX830868 \\
\hline \multirow{2}{*}{$\begin{array}{l}\text { Second largest subunit } \\
\text { gene of RNA } \\
\text { polymerase II (RPB2) }\end{array}$} & bRPB2-6F (c) & TGGGGYATGGTNTGYCCYGC & $56^{\circ} \mathrm{C}, 30 \mathrm{~s}$ & $\mathrm{Cs}$ & 887 & $832(55)$ & KX881864-KX881881 \\
\hline & bRPB2-7.1R (c) & CCCATRGCYTGYTTMCCCATDGC & $\cdots$ & $\mathrm{Cw}$ & 887 & $832(55)$ & KX830869 \\
\hline \multirow{2}{*}{$\begin{array}{l}\text { Translation elongation } \\
\text { factor } 1-\alpha(T E F 1)\end{array}$} & tef1-f (d) & TCMAHGARATYATYAAGGAGAC & $54^{\circ} \mathrm{C}, 45 \mathrm{~s}$ & Cs & 570 & $459(111)$ & KX881882-KX881889 \\
\hline & tef1-rc (d) & DGGGTCGTTYTTSGAGTCA & $\cdots$ & $\mathrm{Cw}$ & 570 & $459(111)$ & KX830858-KX830859 \\
\hline \multirow[t]{2}{*}{$\operatorname{Actin}(A C T 2)$} & psppact2-38f (e) & TGGAACAACGCCTCGGG & $55^{\circ} \mathrm{C}, 30 \mathrm{~s}$ & Cs & $623-626$ & $574(49-52)$ & KX881818-KX881834 \\
\hline & psppact2-701rc (e) & CAGTCGAAGCGYGGTATCTTGAC & $\ldots$ & $\mathrm{Cw}$ & 623 & $574(49)$ & KX830860-KX830863 \\
\hline \multirow{2}{*}{$\begin{array}{l}\text { Laccase N-terminal } \\
\text { copper-binding region }\end{array}$} & pslaccase-15f (e) & YTTCCAAGCCGGAACAAAC & $52^{\circ} \mathrm{C}, 30 \mathrm{~s}$ & $\mathrm{Cs}$ & $115-116$ & $59(56-57)$ & KX881890-KX881903 \\
\hline & pslaccase-170rc (e) & GGGGACGCTAAAGTCGTATCT & $\ldots$ & $\mathrm{Cw}$ & 115 & $59(56)$ & KX830864 \\
\hline
\end{tabular}

y References: $\mathrm{a}=$ White et al. (1990), $\mathrm{b}=$ Vilgalys and Hester (1990), $\mathrm{c}=$ Matheny (2005), $\mathrm{d}=$ Bergeron et al. (2011), and e = this study

${ }^{\mathrm{z}} \mathrm{Cs}=$ Coniferiporia sulphurascens and $\mathrm{Cw}=C$. weirii. 
Eurasian population had greater diversity than the NA population, with 14 distinct MLGs observed among the16 isolates genotyped (Fig. 1B; Table 1). The Eurasian population also had higher haplotypic diversity (67 of 97 haplotypes) than the NA population (30 of 97 haplotypes). Findings were similar when considering only the five protein-coding genes: we identified 47 distinct haplotypes within the Eurasian population resulting in an average haplotypic diversity value of $0.77 \pm 0.09$ ( 0.59 for the $R P B 2$ gene to 0.91 for ACT2) (Table 3). Haplotypic diversity was more than half lower in the NA population (average of $0.31 \pm 0.16$; ranging from 0.08 for $T E F 1$ to 0.58 for $L A C$ ), with only 24 distinct haplotypes (Table 3; Supplementary Table S2). The same trend was observed for the genetic diversity, with the number of segregating sites more than half lower in NA than in Eurasia (33 sites in NA compared with 85 in Eurasia across a sequence alignment of 2,770 bp) and an average nucleotidic diversity four times lower $(0.012 \pm 0.002$ in NA compared with $0.047 \pm 0.009$ in Eurasia). Finally, most variants were fixed in NA and polymorphic in Eurasia $(n=88)$, with $68.8 \%$ of SNPs and indels observed within the nuclear loci (Table 4).

Population structure of $C$. sulphurascens. At $K=2$, the Bayesian clustering algorithm assigned NA samples to one cluster and those from Eurasia to a second cluster. At $K=3$, NA individuals originating from the same region were not sharing similar membership coefficients, with the exception of individuals from the IDF zone in British Columbia and Montana, indicating the absence of a clear geographic structure. The STRUCTURE analysis on the 16 Eurasian isolates also failed to reveal any clusters within this population, based on their geographic origin (Fig. 2).

Similarly, an analysis of molecular variance indicated that most of the genetic variation could be explained by differences between populations on the two continents $(76 \%)$ rather than by variation among geographic origins within continents and within individuals (3 and 21\%, respectively) (Supplementary Table S4). The degree of genetic differentiation between these two continental populations was also contrasted against other species by using the average number of nucleotide substitutions per site between populations (Dxy parameter). Differentiation between the NA and Eurasian populations is far below the degree of differentiation observed between $C$. sulphurascens and $C$. weirii or $C$. sulphurascens and Phellinus noxius (Supplementary Fig. S1), in accordance with the divergence time estimations (outlined below).

We examined the influence of landscape features on shaping the NA population of $C$. sulphurascens by PCoA, which showed three main groups (Fig. 3A). However, this PCoA analysis did not produce any clear grouping of isolates on the basis of sites in NA or biogeoclimatic zones in British Columbia, with the exception of the IDF biogeoclimatic zone, which formed a distinct group, as previously observed with the STRUCTURE analysis. It is interesting to note that, of the four biogeoclimatic zones from British Columbia used in this study, IDF is much drier and cooler compared with the other three biogeoclimatic zones (Reynolds 1992). The segregation of isolate T-124 (collected from Quilcine, WA) could be explained by its particularity of being the only isolate in the homozygous state for

\section{North America}

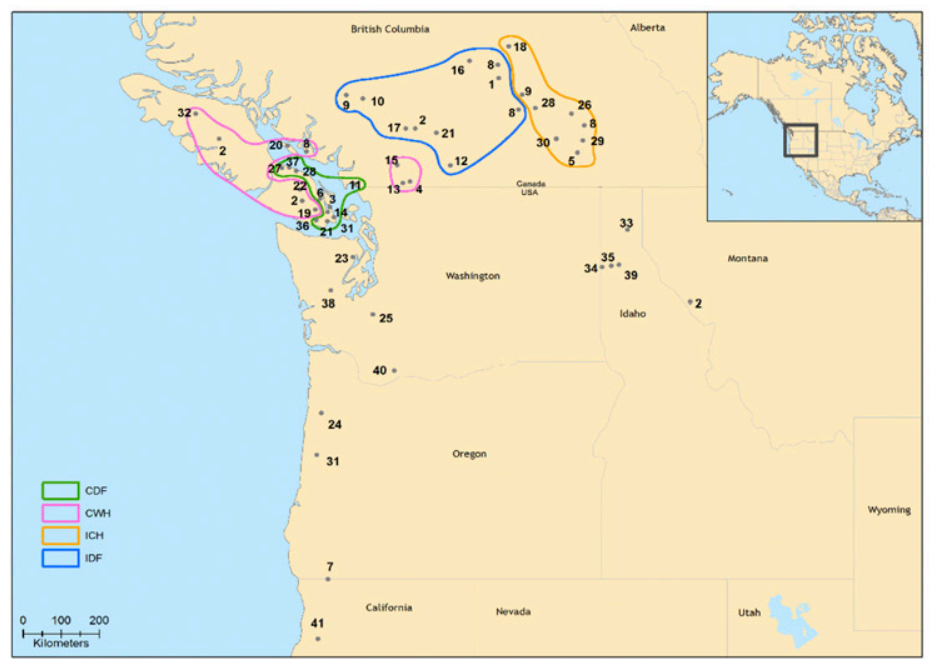

\section{Eurasia}

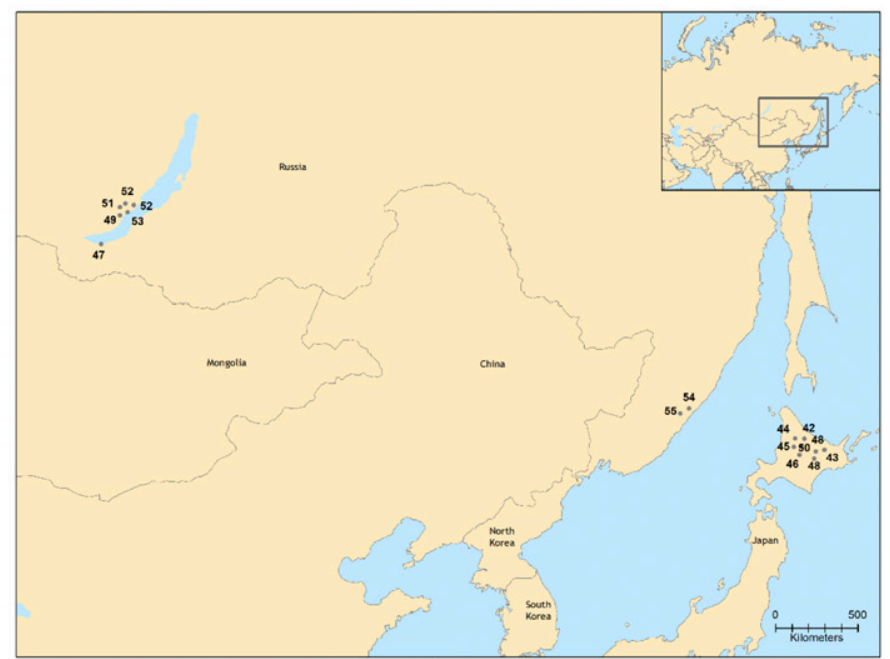

Fig. 1. Geographic location of Coniferiporia sulphurascens isolates in North America, with the four biogeoclimatic zones outlined, and Eurasia. Each number represents a unique multilocus genotype (MLG), based on two mitochondrial ribosomal RNA genes (mtSSU rRNA and mtLSU rRNA), five nuclear protein-coding genes $(A C T 2, R P B 1, R P B 2, L A C$, and $T E F 1)$, and the internal transcribed spacer region. $\mathrm{CDF}=\mathrm{Coastal}$ Douglas-fir, $\mathrm{CWH}=\mathrm{Coastal}$ Western Hemlock, ICH $=$ Interior Cedar-Hemlock, and IDF = Interior Douglas-fir.

TABLE 3. Molecular diversity parameters at five nuclear protein-coding genes sequenced in Coniferiporia sulphurascens ${ }^{y}$

\begin{tabular}{|c|c|c|c|c|c|c|c|c|c|}
\hline \multirow[b]{2}{*}{ Locus $^{\mathrm{z}}$} & \multicolumn{3}{|c|}{ Eurasia $(n=16)$} & \multicolumn{3}{|c|}{ North America $(n=58)$} & \multicolumn{3}{|c|}{ Total $(n=74)$} \\
\hline & $\mathrm{S}$ & $\mathrm{H}\left(\mathrm{H}_{\mathrm{d}}\right)$ & $\mathrm{K}\left(\pi 10^{-3}\right)$ & $S$ & $\mathrm{H}\left(\mathrm{H}_{\mathrm{d}}\right)$ & $\mathrm{K}\left(\pi 10^{-3}\right)$ & $S$ & $\mathrm{H}\left(\mathrm{H}_{\mathrm{d}}\right)$ & $\mathrm{K}\left(\pi 10^{-3}\right)$ \\
\hline ACT2 & 29 & $12(0.91)$ & $5.72(9)$ & 14 & $5(0.18)$ & $1.6(3)$ & 41 & $17(0.49)$ & 4.67 (7) \\
\hline$R P B 1$ & 21 & $10(0.84)$ & 3.59 (6) & 3 & $4(0.25)$ & $0.25(0.44)$ & 28 & $14(0.53)$ & 3.77 (7) \\
\hline$L A C$ & 11 & $9(0.73)$ & $2.81(24)$ & 4 & $5(0.58)$ & $0.65(6)$ & 16 & $14(0.73)$ & $2.12(18)$ \\
\hline TEF1 & 6 & $5(0.76)$ & $2.36(4)$ & 2 & $3(0.08)$ & $0.08(0.15)$ & 8 & $8(0.43)$ & $0.76(1)$ \\
\hline
\end{tabular}

y $\mathrm{S}=$ number of segregating sites. Haplotypic diversity: $\mathrm{H}=$ number of haplotypes and $\mathrm{H}_{\mathrm{d}}=$ index of haplotype diversity. Genetic diversity: $\mathrm{K}=$ average number of nucleotide differences and $\pi=$ nucleotide diversity per site.

${ }^{\mathrm{z}} A C T 2=$ actin, $R P B 1=$ RNA polymerase II largest subunit, $R P B 2=$ RNA polymerase II second-largest subunit, $L A C=$ laccase-like multicopper oxidase, and $T E F 1=$ translation elongation factor $1-\alpha$. 
the $R P B 2$ gene with the genotype $\mathrm{CC}$ (Table 1 ). The influence of host species was also examined but no clear clusters of isolates were observed in the NA population based on hosts (Fig. 3B and C). From the results obtained by PCoA for the Eurasian population, two major clusters were found. One of these two groups was composed of six Siberian isolates, all collected on Picea abies subsp. obovata in the vicinity of Lake Baikal (Fig. 3B and D). However, because P. abies subsp. obovata was only sampled within the region surrounding Lake Baikal, the extent to which geographic location or host might influence the population shaping cannot be clearly established.

Reproductive mode within $C$. sulphurascens populations. Significant linkage disequilibrium was observed within the NA and Eurasian populations, with linkage persisting in clone-corrected samples $\left(\mathrm{I}_{\mathrm{A}}\right.$ and $\left.\bar{r}_{\mathrm{d}}>0.0 ; P<0.01\right)$ (Table 5). This supports the hypothesis of predominant clonal evolution structure throughout vegetative spread or inbreeding, rather than an occasional spread of clonal haplotypes in a basically sexual species (Smith et al. 2000). In addition, a strong difference in the degree of clonality was observed between the two populations, with a linkage disequilibrium less pronounced in the Eurasian population (clone-corrected $\mathrm{I}_{\mathrm{A}}=$ $\left.2.90 ; \bar{r}_{\mathrm{d}}=0.04\right)$ than in the NA one $\left(\mathrm{I}_{\mathrm{A}}=4.86 ; \bar{r}_{\mathrm{d}}=0.17\right)$ (Table 5).

Genealogies of $\boldsymbol{C}$. sulphurascens. The concatenated dataset of the five protein-coding genes and ITS region resulted in 75 singleton variable sites and 56 parsimony-informative characteristics over 3,214 bp analyzed (Supplementary Table S3). This combined dataset resulted in similar ML and Bayesian tree topologies that placed C. sulphurascens isolates from NA and Eurasia on different branches (bootstrap [BS] value $=81 \%$ and posterior probability $[\mathrm{PP}]$ value $=1.0)($ Fig. 4). Topology of this combined phylogeny was consistent with four of the single-gene tree partitions ( $A C T 2, R P B 1, R P B 2$, and $L A C)$, resolving $C$. sulphurascens isolates into two geographic clades. The ITS region and the TEF1 gene were not informative enough to resolve the phylogenetic partition into the two clades (Supplementary Fig. S2).

To investigate the extent of differentiation of the NA population from the Eurasian population, we inferred the phylogenetic relationship among the alleles of five protein-coding genes with $C$. weirii, Phellinus ferrugineofuscus, $P$. lamaoensis, and $P$. noxius as outgroups (Fig. 5). Although all the alleles of the NA and Eurasia populations shared a common ancestor, only alleles of the NA isolates in RPBI and $L A C$ clustered all together and evolved independently from alleles of the Eurasian isolates.

Divergence time estimation of the NA from the Eurasian populations of $\boldsymbol{C}$. sulphurascens. Divergence time estimates indicated that the Hymenochaetales order radiated 225 Mya (125 to 338 Mya, 95\% HPD) during the early Triassic and C. sulphurascens would have diverged from $C$. weirii approximately 38 Mya (14.0

TABLE 4. Fixed and shared polymorphisms between Coniferiporia sulphurascens isolates from North America (NA) and Eurasia

\begin{tabular}{|c|c|c|c|c|}
\hline \multirow[b]{2}{*}{ Locus $^{\mathrm{z}}$} & \multicolumn{3}{|c|}{ Fixed polymorphisms ${ }^{\mathrm{y}}$} & \multirow{2}{*}{$\begin{array}{c}\text { Shared polymorphism } \\
\begin{array}{c}\text { Polymorphic in NA } \\
\text { and Eurasia }\end{array}\end{array}$} \\
\hline & Fixed in NA and Eurasia & $\begin{array}{l}\text { Fixed in NA and polymorphic } \\
\text { in Eurasia }\end{array}$ & $\begin{array}{l}\text { Fixed in Eurasia and } \\
\text { polymorphic in NA }\end{array}$ & \\
\hline$A C T 2$ & 0 & $27 *$ & $12 *$ & 2 \\
\hline$L A C$ & 2 & $10 *$ & 3 & 1 \\
\hline$R P B 1$ & 4 & 21 & 3 & 0 \\
\hline$R P B 2$ & 0 & 17 & 9 & 1 \\
\hline$T E F 1$ & 0 & 6 & 2 & 0 \\
\hline ITS & 0 & 7 & 1 & 0 \\
\hline Total & 6 & 88 & 30 & 4 \\
\hline SNP $(\%)$ & 4.7 & 68.8 & 23.4 & 3.1 \\
\hline
\end{tabular}

y An asterisk $(*)$ indicates including indels.

z $A C T 2=$ actin, $L A C=$ laccase-like multicopper oxidase, $R P B 1=$ RNA polymerase II largest subunit, $R P B 2=$ RNA polymerase II second-largest subunit, $T E F 1=$ translation elongation factor $1-\alpha$, ITS = internal transcribed spacer, and SNP = single nucleotide polymorphism.
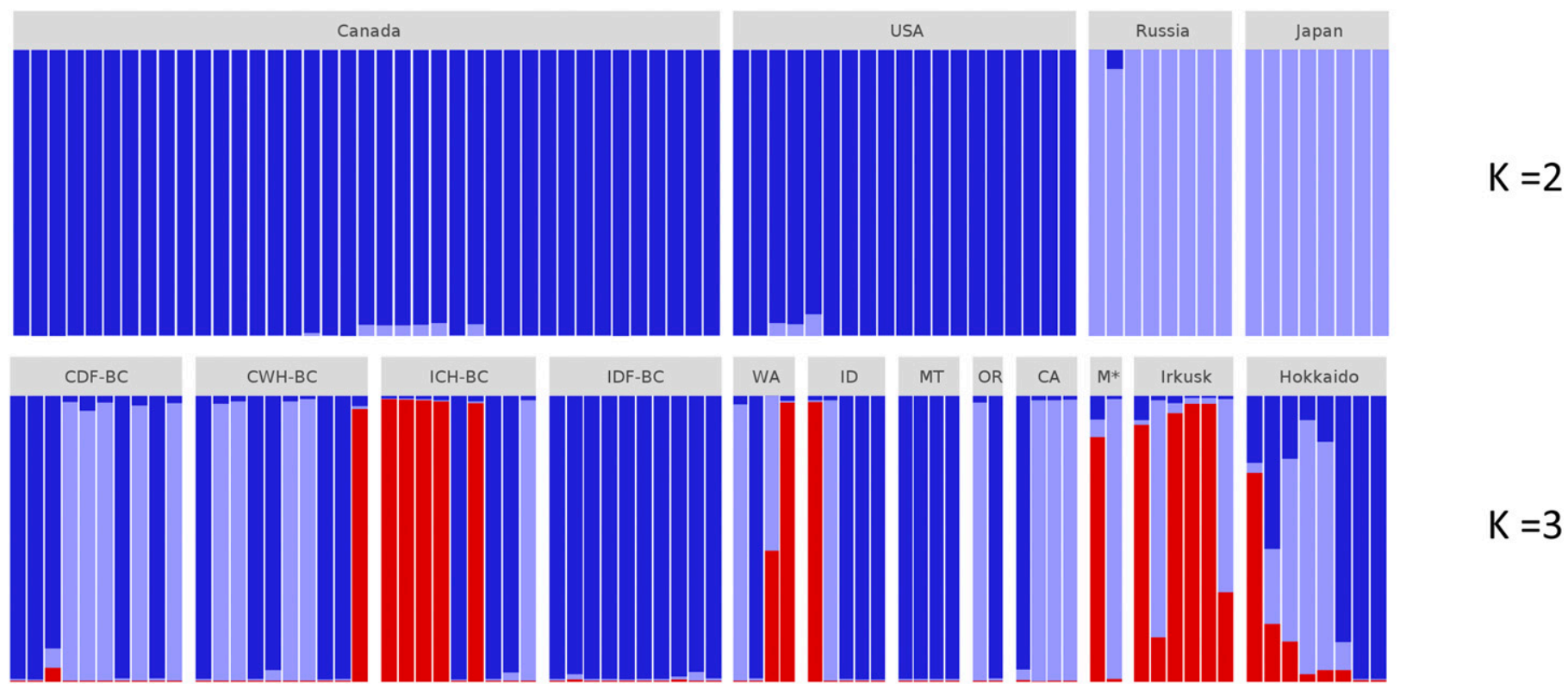

Fig. 2. Population structure of Coniferiporia sulphurascens as inferred by STRUCTURE. Top: North American and Eurasian populations. Bottom: Regions within North America and Eurasia. Each individual is represented by a vertical bar, showing the proportions of membership to the different genetic clusters. CDF = Coastal Douglas-fir, $\mathrm{CWH}=$ Coastal Western Hemlock, ICH = Interior Cedar-Hemlock, and IDF = Interior Douglas-fir; WA = Washington, ID = Idaho, MT = Montana, $\mathrm{OR}=$ Oregon, and CA $=$ California states in the United States; and the asterisk $(*)$ refers to Maise in Russia. 
to 66.0 Mya, 95\% HPD). The NA population of $C$. sulphurascens would have diverged from the Eurasian one approximately 7.5 Mya (2.6 to 13.3 Mya, 95\% HPD), at the end of the Miocene period (Fig. 6).

\section{DISCUSSION}

Here, we present a multilocus genotyping study exploring genetic population structure and cryptic species diversity within C. sulphurascens from NA and Eurasia based on the phylogenetic species recognition approach (Taylor et al. 2000). Data analyses of two mitochondrial and six nuclear loci strongly supported the existence of a species complex within $C$. sulphurascens with at least two highly differentiated genetic lineages. A global population structure analysis indicated a considerable degree of differentiation between the two continental populations, reflecting their geographical isolation. The concatenated six-gene phylogeny as well as the genealogies of isolates in single-gene phylogeny $(A C T 2$, $R P B 1, R P B 2$, and $L A C$ ) clearly divided the NA and Eurasian populations into two distinct clades, suggesting that the NA population could represent a cryptic species that diverged from the Eurasian population. However, single-gene phylogenies such as
ITS and TEF1 did not resolve NA and Eurasian isolates into two separate clades. This is probably the result of incomplete lineage sorting, and it suggests that these two populations are closely related and still diverging from each other. In some cases, combined phylogeny analyses with additional genes can be more informative and powerful than single-gene analysis regarding the resolution of internal branches (Feau et al. 2006; Gadagkar et al. 2005; Gontcharov et al. 2004).

TABLE 5. Indexes of association $\left(\mathrm{I}_{\mathrm{A}}\right.$ and $\bar{r}_{\mathrm{d}}$ ) values for Coniferiporia sulphurascens populations

\begin{tabular}{lccc}
\hline Population & $\begin{array}{c}\text { Number of } \\
\text { individuals }\end{array}$ & $\mathrm{I}_{\mathrm{A}}(P$ value $)$ & $\bar{r}_{\mathrm{d}}(P$ value $)$ \\
\hline $\begin{array}{l}\text { North America } \\
\text { North America }\end{array}$ & 58 & $5.39(0.001)$ & $0.20(0.001)$ \\
$\quad$ (clone-corrected) & 33 & $4.86(0.001)$ & $0.17(0.001)$ \\
$\quad \begin{array}{l}\text { Eurasia } \\
\text { Eurasia (clone- }\end{array}$ & 16 & $3.54(0.001)$ & $0.05(0.001)$ \\
$\quad$ corrected) & 14 & $2.90(0.001)$ & $0.04(0.001)$ \\
All & 74 & $23.31(0.001)$ & $0.28(0.001)$ \\
All (clone-corrected) & 47 & $20.02(0.001)$ & $0.23(0.001)$ \\
\hline
\end{tabular}
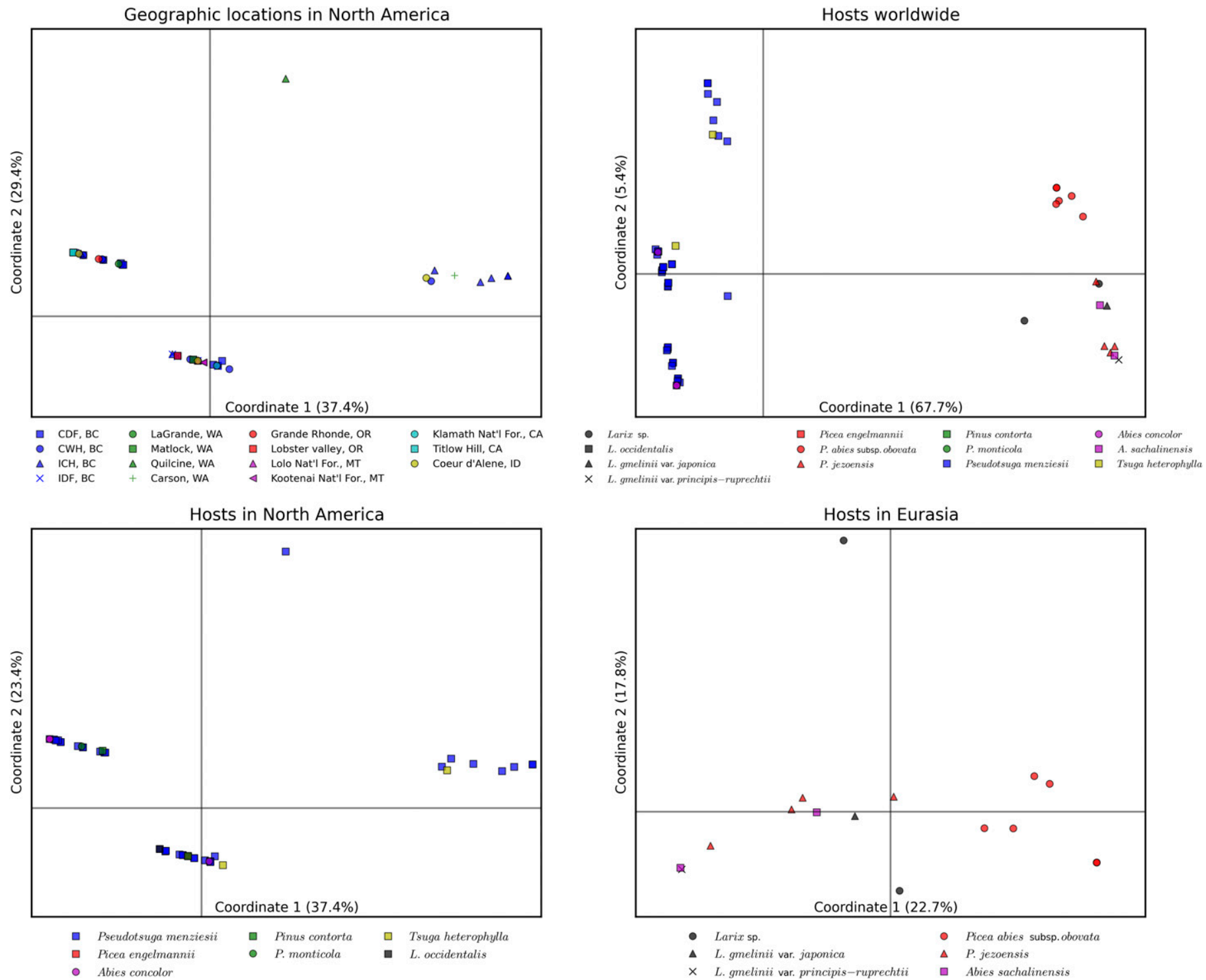

Fig. 3. Principal coordinate analysis of Coniferiporia sulphurascens isolates, according to geographic location in North America, hosts worldwide, hosts in North America, and hosts in Eurasia. First and second coordinates account for 37.4 and $29.4 \%$ of the total genetic diversity in North America, 67.7 and $5.4 \%$ in hosts worldwide, 37.4 and 23.4\% in hosts in North America, and 22.7 and 17.8\% in hosts in Eurasia. CDF $=$ Coastal Douglas-fir, CWH $=$ Coastal Western Hemlock, $\mathrm{ICH}=$ Interior Cedar-Hemlock, and IDF = Interior Douglas-fir. 
Higher haplotype and genetic diversities were observed in the Eurasian population as measured by diversity indexes that take into account the relative abundance of each population. This higher diversity found in Eurasia and the fact that a few alleles in the NA population appeared to have evolved from within the Eurasian population suggest that the Eurasian population could be ancestral to the NA population. The analysis of reproductive mode also supports this hypothesis. The NA population had a higher degree of linkage disequilibrium than the Eurasian population, suggesting that vegetative spread or inbreeding may play a more important role in shaping the genetic structure of the NA population. In addition, limited sexual reproduction in this population could be the result of a founder event, with a reduced effective population size resulting in unbalanced frequencies of
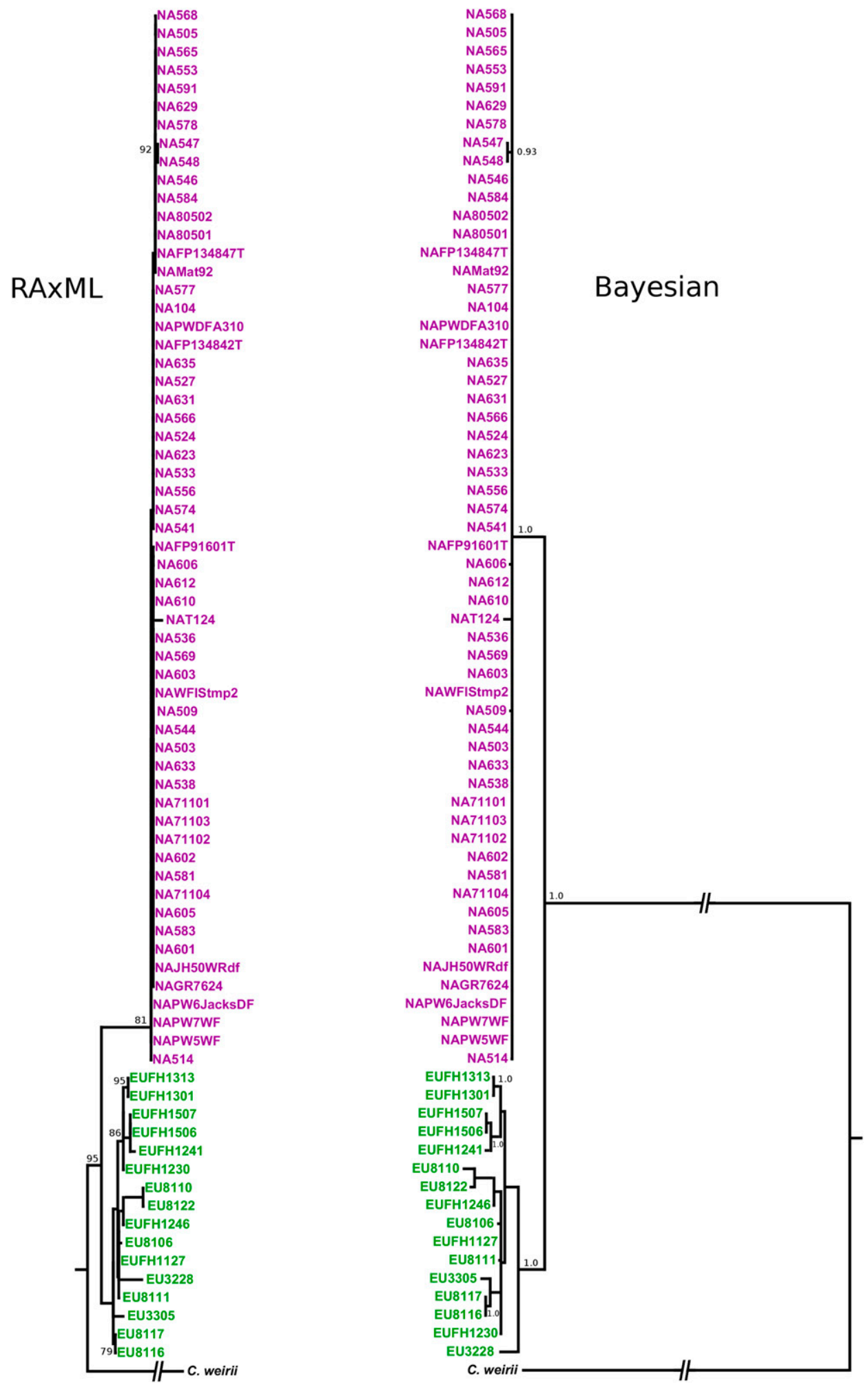

Fig. 4. Best-scoring maximum-likelihood (ML) and Bayesian analysis trees for the combined ACT2, LAC, RPB1, RPB2, TEF1, and ITS datasets, with $\geq 70 \%$ bootstrap values shown above the supported branches. The $50 \%$ majority rule consensus tree, based on B/MCMC (Bayesian/Monte Carlo Markov Chain) phylogenetic inferences for the combined datasets with $\geq 0.90$ posterior probability values, are shown above the branches. Isolates from North America are shown in purple and isolates from Eurasia are shown in green. Coniferiporia weirii was used as an outgroup taxon. 
the two mating type idiomorphs, which has been observed in other plant pathogens (Brasier 2001; Goodwin et al. 1994; Santana et al. 2016).

The Eurasian population might constitute a diversity center in which more lineages of $C$. sulphurascens have evolved compared with the NA population. The NA-derived population has low levels of genetic diversity, possibly due to a genetic drift effect following its founding population and to a limited host range in NA compared with Eurasia. There is little evidence to suggest that the NA population has coevolved with the endemic hosts. Instead, this population could have adapted to some different and lower number of host species than the ancestral Eurasian population. Such host specializations driving fungal speciation have been reported in several tree root rot pathogens from the genus Heterobasidion (Chen et al. 2015) and many other fungal phytopathogens (Giraud et al. 2010).

Intercontinental separation is the major factor contributing to the divergence of the NA population from the Eurasian population. It is likely that the NA cryptic species diverged primarily as a result of geographic isolation rather than host specialization. Such intercontinental divergence has been observed in other forest pathogens (Chen et al. 2015; Song and Cui 2017; Song et al. 2016). Our divergence time estimation data suggest that the NA population diverged from the Eurasian population approximately 7.5 Mya. The ancestral population of $C$. sulphurascens could have expanded from northeastern China and eastern Russia (Eurasia) into the western part of NA via the Bering Land Bridge, which was open for
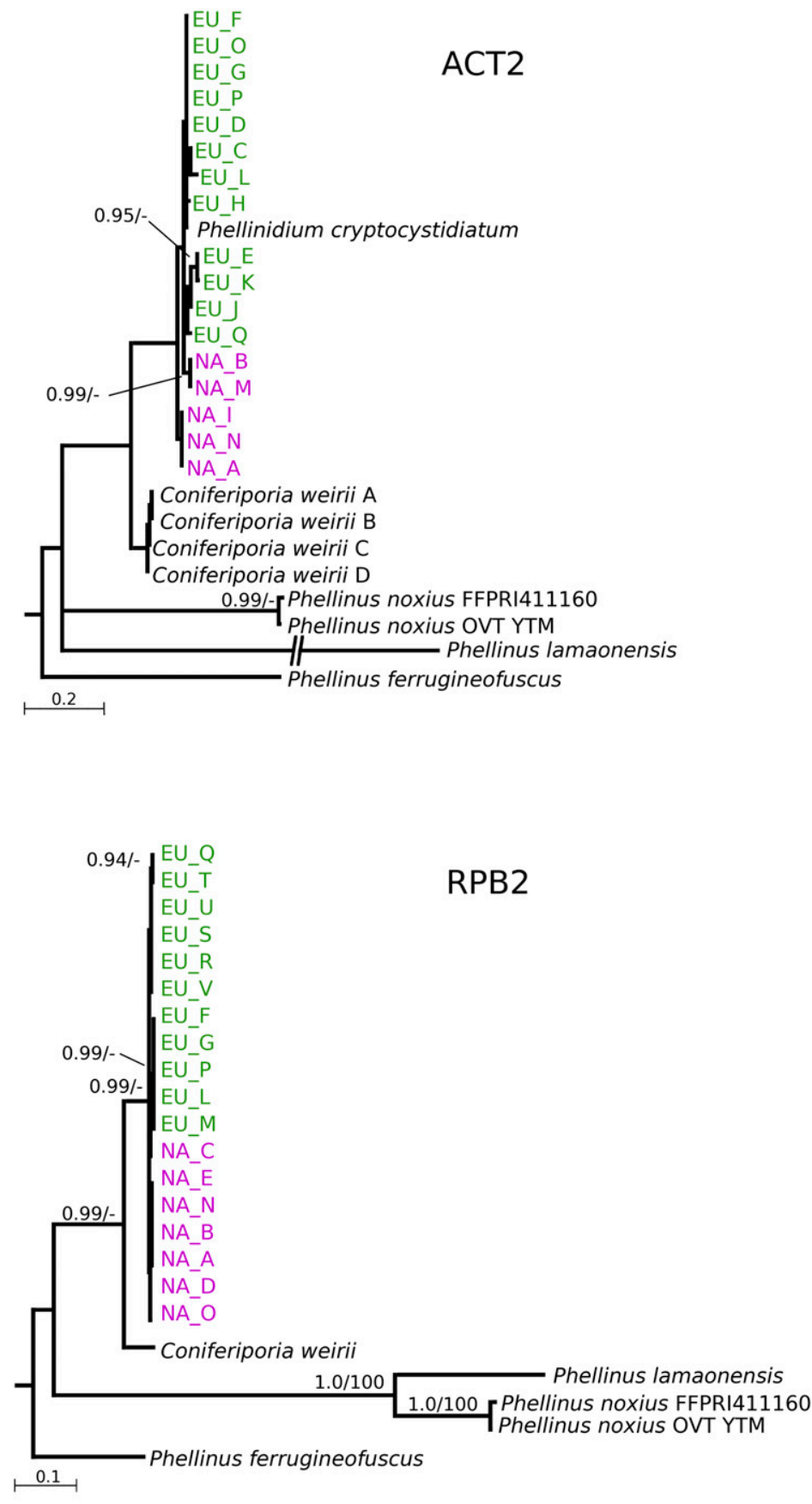
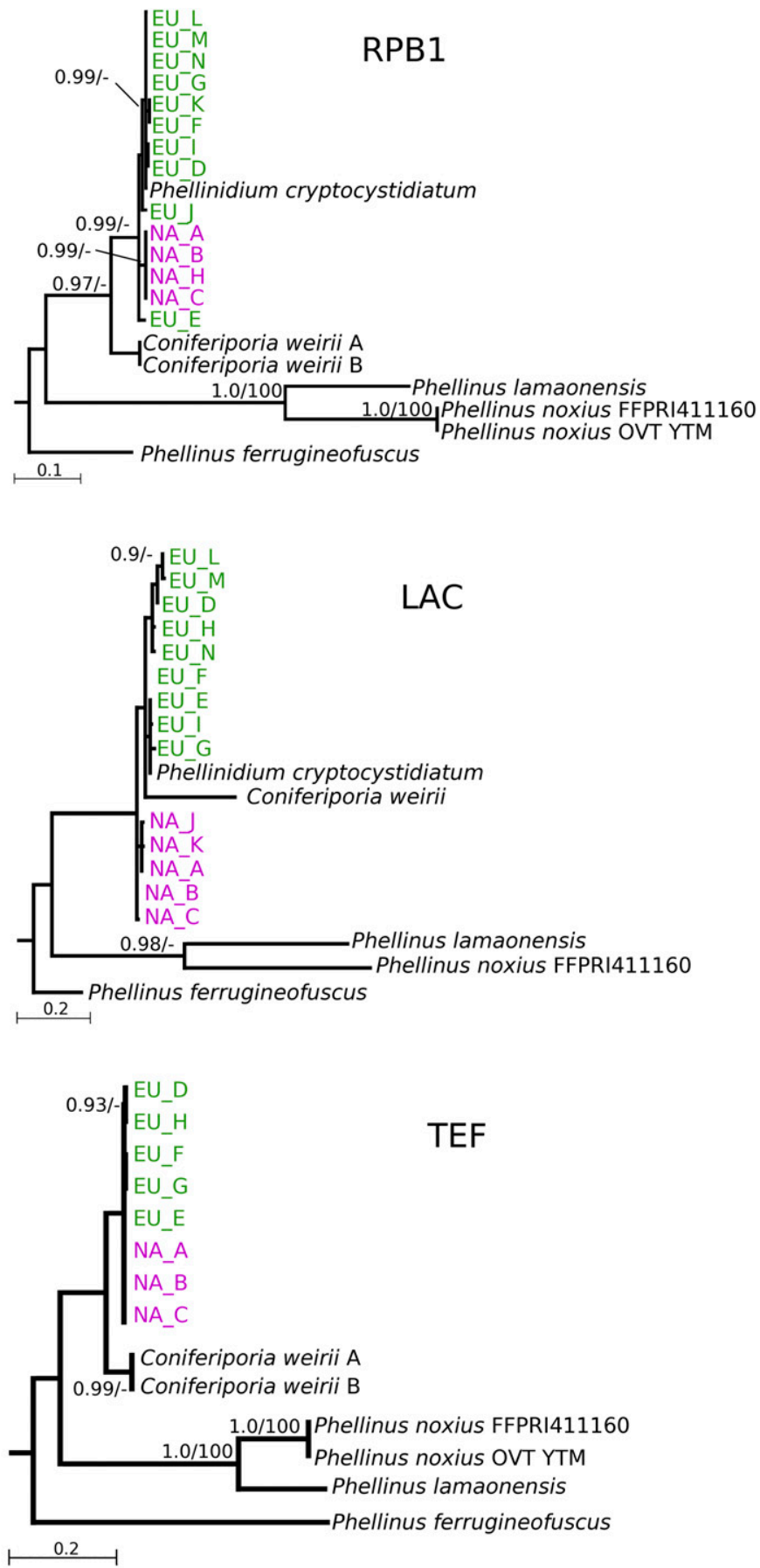

Fig. 5. Phylogenetic relationship among the alleles of five protein-coding genes (ACT2, LAC, RPB1, RPB2, and TEF1) genotyped in Coniferiporia sulphurascens. Statistical support indicated the neighbor-joining bootstrap values. Isolates from North America are shown in purple and isolates from Eurasia are shown in green. C. weirii was used as an outgroup taxon. 
floral and faunal exchange in the Palaearctic and Nearctic forests between 5.4 to 5.5 and 100 Mya (Gladenkov et al. 2002; Graham 1999). Environmental change and spatial isolation at the Bering Strait opening (end of the Miocene) and during the following Ice Age (Pleistocene) beginning 2.58 Mya may have driven the speciation of the NA population. Similarly, Heterobasidion spp. radiated at 19.72 Mya and the NA representative Heterobasidion irregulare, a forest root pathogen, diverged approximately 10 Mya, owing to migration from Eurasia into northwestern America via the Bering Land Bridge (Chen et al. 2015). Limited genetic diversity observed in the NA population (relative to the Eurasian population) suggests that genetic drift might have occurred in this population due to limited gene flow and reduction in effective population size (i.e., founder effect). In parallel, the possibility of different selective pressures exerted onto the two populations cannot be ruled out, given the differences in host diversity and climatic conditions observed between the NA and Eurasian regions. Modeling of the demography history of the global $C$. sulphurascens population should help to answer these questions.

Even though the NA and Eurasian populations represent two different lineages, mating compatibility between $C$. sulphurascens isolates from NA and Eurasia has been observed (Angwin and Hansen 1993; Hansen et al. 1998; Lim et al. 2008). According to Taylor et al. (2000), fungal speciation studies show that, among allopatric sibling species, genetic divergence may have occurred before these species lost their ability to mate with one another.

In 2016, Zhou et al. (2016) introduced and proposed the new genus Coniferiporia, which segregates from the genus Phellinidium three forest pathogens: $C$. sulphurascens, $C$. weirii, and $C$. qilianense. These authors did not use any NA isolates of the $C$. sulphurascens complex in their phylogenetic analysis, based on the $n L S U$ gene. Nonetheless, the results that we report here, based on genes that resolve deeper relationships than does $n L S U$, leave no doubt that the NA lineage should be included in this new genus. Moreover, our preliminary findings on a herbarium specimen of Phellinidium cryptocystidiatum from Russia support and refine the observations made by Zhou et al. (2016), who defined this fungus as a synonym of $C$. sulphurascens. Indeed, based on $A C T 2, R P B 1$, and $L A C$ gene sequencing, we found that $P$. cryptocystidiatum shares the same alleles with the Eurasian C. sulphurascens isolates used in this study (Fig. 5).

In conclusion, the genetic diversity study of $C$. sulphurascens isolates from widespread locations in British Columbia, the northwestern United States, and Eurasia (Siberia and Japan) demonstrated the presence of a cryptic lineage; the clonal NA population is well differentiated from the highly diverse Eurasian population. The genetic divergence between the two lineages is possibly linked to ancient dispersal via the Bering Land Bridge between the two continents. Although it is difficult to compare the pathology and ecology that characterize these two populations due to limited availability of relevant data for C. sulphurascens in Eurasia (Dai and Qin, 1998), they should be recognized as two distinct sibling species, based on the criteria of the phylogenetic species recognition (Taylor et al. 2000). The Eurasian lineage could retain the name $C$. sulphurascens, because this fungus was originally described from the Primorsk Territory, Russia (Larsen et al. 1994), while the NA lineage should be recognized as a new species.

Our data should facilitate further investigation of whether the NA and Eurasian lineages can be recognized as two separable species

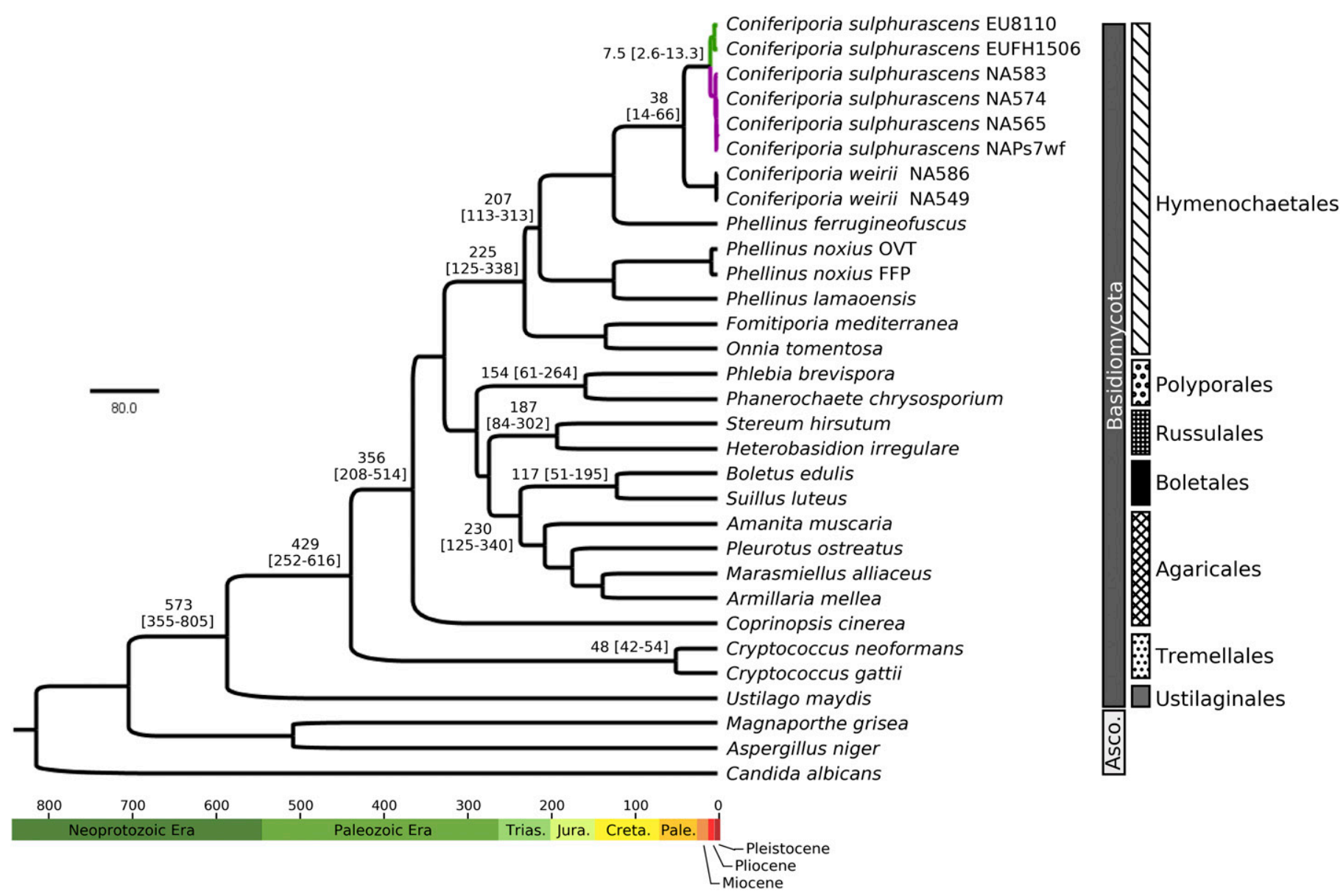

Fig. 6. Divergence time estimation of Coniferiporia sulphurascens and other species of Basidiomycetes based on molecular clock analyses using four nuclear protein-coding genes (ACT2, RPB1, RPB2, and TEF1) by BEAST. Values at main nodes indicate the divergence time in millions of years followed by $95 \%$ highest posterior densities. 
based on morphological, physiological, and pathological characteristics. We will undertake further morphological analysis to identify informative characteristics that correlate with the two phylogenetic species.

\section{ACKNOWLEDGMENTS}

We thank K. Korhonen (Finnish Forest Research Institute, Finland); B. Lockman, S. Hagle, and P. Anwin (United States Department of Agriculture Forest Service); T. Yamaguchi (Hokkaido Research Centre, Japan); and The Centre for Forest Mycology Research (Madison, WI, USA) for kindly providing the isolates used in this study; V. Spirin (Botany Unit Finnish Museum of Natural History, Finland) for sending us herbarium specimen samples of $P$. cryptocystidiatum; and G. Thandi (Pacific Forestry Centre, Canada) for mapping the NA and Eurasian isolates.

\section{LITERATURE CITED}

Agapow, P. M., and Burt, A. 2001. Indices of multilocus linkage disequilibrium. Mol. Ecol. Resour. 1:101-102.

Akiba, M., Ota, Y., Tsai, I. J., Hattori, T., Sahashi, N., and Kikuchi, T. 2015. Genetic differentiation and spatial structure of Phellinus noxius, the causal agent of brown root rot of woody plants in Japan. PLoS One 10:e0141792.

Angwin, P., and Hansen, E. 1993. Pairing tests to determine mating compatibility in Phellinus weirii. Mycol. Res. 97:1469-1475.

Aoshima, K. 1953. Wood-rotting Poria from Japan. Bull. Gov. For. Exp. Stn. (Ringyo Shikenjo kenkyu hokoku) 59:57-64.

Baumgartner, K., Travadon, R., Bruhn, J., and Bergemann, S. E. 2010. Contrasting patterns of genetic diversity and population structure of Armillaria mellea sensu stricto in the eastern and western United States. Phytopathology 100:708-718.

Beale, J. D. 1992. Laminated root rot: Ecology relationship and stand productivity impacts in coastal Douglas-fir ecosystems of British Columbia. M.S. thesis, University of British Columbia, Vancouver, BC, Canada.

Berbee, M. L., and Taylor, J. W. 2010. Dating the molecular clock in fungihow close are we? Fungal Biol. Rev. 24:1-16.

Bergeron, M.-J., Leal, I., Foord, B., Ross, G., Davis, C., Slippers, B., De Groot, P., and Hamelin, R. C. 2011. Putative origin of clonal lineages of Amylostereum areolatum, the fungal symbiont associated with Sirex noctilio, retrieved from Pinus sylvestris, in eastern Canada. Fungal Biol. 115: 750-758.

Brasier, C. M. 2001. Rapid evolution of introduced plant pathogens via interspecific hybridization: hybridization is leading to rapid evolution of Dutch elm disease and other fungal plant pathogens. A.I.B.S. Bull. 51: 123-133.

Buckland, D. C., Molnar, A. C., and Wallis, W. G. 1954. Yellow laminated root rot of Douglas-fir. Can. J. Bot. 32:69-81.

Burdon, J. J. 1993. Genetic variation in pathogen populations and its implications for adaptation to host resistance. Pages 41-56 in: Durability of Disease Resistance, Vol. 18. T. Jacobs and J. E. Parlevliet, eds. Springer Netherlands, Dordrecht The Netherlands.

Burdon, J. J., and Silk, J. 1997. Sources and patterns of diversity in plant pathogenic fungi. Phytopathology 87:664-669.

Campanile, G., Giove, S. L., and Luisi, N. 2004. Genetic and morphologic variability of Phellinus torulosus isolates in some oak woods of Southern Italy. J. Plant Pathol. 86:105-115.

Chen, J.-J., Cui, B.-K., Zhou, L.-W., Korhonen, K., and Dai, Y.-C. 2015. Phylogeny, divergence time estimation, and biogeography of the genus Heterobasidion (Basidiomycota, Russulales). Fungal Divers. 71: 185-200.

Chung, C.-L., Lee, J. T., Akiba, M., Lee, H.-H., Kuo, T.-H., Liu, D., Ke, H.-M., Yokoi, T., Roa, M. B., and Lu, M. J. 2017. The genomic landscape of tree rot in Phellinus noxius and its hymenochaetales members. bioRxiv Article 132712

Cook, R. J., Edmonds, R. L., Klopfenstein, N. B., Littke, W., McDonald, G., Omdahl, D., Ripley, K., Shaw, C. G., Sturrock, R., and Zambino, P. 2013. Opportunities for Addressing Laminated Root Rot Caused by Phellinus sulphuracens in Washington's Forests: A Report from the Washington State Academy of Sciences in Cooperation with the Washington State Department of Natural Resources. Washington State Academy of Sciences, Olympia, WA.

Cooke, D. E. L., Jung, T., Williams, N. A., Schubert, R., Osswald, W., and Duncan, J. M. 2005. Genetic diversity of European populations of the oak fine-root pathogen Phytophthora quercina. For. Pathol. 35:57-70.

Dai, Y.-C. 2010. Hymenochaetaceae (Basidiomycota) in China. Fungal Divers. 45:131-343.
Dai, Y.-C., and Qin, G.-F. 1998. Phellinidium sulphurascens-a forest pathogen in China. Fungal Sci. 13:101-107.

Dalman, K., Olson, ^., and Stenlid, J. 2010. Evolutionary history of the conifer root rot fungus Heterobasidion annosum sensu lato. Mol. Ecol. 19: 4979-4993.

Drummond, A. J., and Rambaut, A. 2007. BEAST: Bayesian evolutionary analysis by sampling trees. BMC Evol. Biol. 7:214.

Earl, D. A., and vonHoldt, B. M. 2012. STRUCTURE HARVESTER: A website and program for visualizing STRUCTURE output and implementing the Evanno method. Conserv. Genet. Resour. 4:359-361.

Feau, N., Hamelin, R. C., and Bernier, L. 2006. Attributes and congruence of three molecular data sets: Inferring phylogenies among Septoria-related species from woody perennial plants. Mol. Phylogenet. Evol. 40: 808-829.

Ferguson, B. A., Dreisbach, T. A., Parks, C. G., Filip, G. M., and Schmitt, C. L. 2003. Coarse-scale population structure of pathogenic Armillaria species in a mixed-conifer forest in the Blue Mountains of northeast Oregon. Can. J. For. Res. 33:612-623.

Gadagkar, S. R., Rosenberg, M. S., and Kumar, S. 2005. Inferring species phylogenies from multiple genes: Concatenated sequence tree versus consensus gene tree. J. Exp. Zoolog. B Mol. Dev. Evol. 304B:64-74.

Garbelotto, M., Guglielmo, F., Mascheretti, S., Croucher, P., and Gonthier, P. 2013. Population genetic analyses provide insights on the introduction pathway and spread patterns of the North American forest pathogen Heterobasidion irregulare in Italy. Mol. Ecol. 22:4855-4869.

Giraud, T., Gladieux, P., and Gavrilets, S. 2010. Linking the emergence of fungal plant diseases with ecological speciation. Trends Ecol. Evol. 25: 387-395.

Gladenkov, A. Y., Oleinik, A. E., Marincovich, L., and Barinov, K. B. 2002. A refined age for the earliest opening of Bering Strait. Palaeogeogr. Palaeoclimatol. Palaeoecol. 183:321-328.

Goheen, D. J., and Hansen, E. M. 1993. Effects of pathogens and bark beetles on forests. Pages 175-196 in: Beetle-Pathogen Interactions in Conifer Forests. T. S. G. Filip, ed. Academic Press, London.

Gontcharov, A. A., Marin, B., and Melkonian, M. 2004. Are combined analyses better than single gene phylogenies? A case study using SSU rDNA and rbcL sequence comparisons in the Zygnematophyceae (Streptophyta). Mol. Biol. Evol. 21:612-624.

Goodwin, S. B., Cohen, B. A., and Fry, W. E. 1994. Panglobal distribution of a single clonal lineage of the Irish potato famine fungus. Proc. Natl. Acad. Sci. USA 91:11591-11595.

Graham, A. 1999. Late Cretaceous and Cenozoic history of North American Vegetation: North of Mexico. Oxford University Press, New York.

Hamelin, R. C., Allaire, M., Bergeron, M.-J., Nicole, M.-C., and Lecours, N. 2005. Molecular epidemiology of white pine blister rust: Recombination and spatial distribution. Phytopathology 95:793-799.

Hansen, E. M. 1979a. Survival of Phellinus weirii in Douglas-fir stumps after logging. Can. J. For. Res. 9:484-488.

Hansen, E. M. 1979b. Sexual and vegetative incompatibility reactions in Phellinus weirii. Can. J. Bot. 57:1573-1578.

Hansen, E. M., Angwin, P. A., Dreisbach, T. A., Gernandt, D., and McWilliams, M. 1998. Species limits for Phellinus weirii. Pages 119-127 in: Root and Butt Rots of Forest Trees: 9th Int. Conf. Root Butt Rots, Carcans-Maubuisson, France. Institut National de la Recherche Agronomique (INRA).

Hansen, E. M., and Goheen, D. J. 2000. Phellinus weirii and other native root pathogens as determinants of forest structure and process in western North America. Annu. Rev. Phytopathol. 38:515-539.

Holah, J. C., Wilson, M. V., and Hansen, E. M. 1993. Effects of a native forest pathogen, Phellinus weirii, on Douglas-fir forest composition in western Oregon. Can. J. For. Res. 23:2473-2480.

Hubisz, M. J., Falush, D., Stephens, M., and Pritchard, J. K. 2009. Inferring weak population structure with the assistance of sample group information. Mol. Ecol. Resour. 9:1322-1332.

Islam, M. A., Sturrock, R. N., and Ekramoddoullah, A. K. M. 2008. A proteomics approach to identify proteins differentially expressed in Douglas-fir seedlings infected by Phellinus sulphurascens. J. Proteomics 71:425-438.

Islam, M. A., Sturrock, R. N., and Ekramoddoullah, A. K. M. 2013. Gene expression profiling of a compatible interaction between Douglas-fir and the root rot fungal pathogen Phellinus sulphurascens. Phytopathology 103:583-593.

Islam, M. A., Sturrock, R. N., Williams, H. L., and Ekramoddoullah, A. K. M. 2010. Identification, characterization, and expression analyses of class II and IV chitinase genes from Douglas-fir seedlings infected by Phellinus sulphurascens. Phytopathology 100:356-366.

Kamvar, Z. N., Tabima, J. F., and Grünwald, N. J. 2014. Poppr: An R package for genetic analysis of populations with clonal, partially clonal, and/or sexual reproduction. PeerJ 2:e281. 
Kastner, W. W., Jr., Goheen, D. J., and Edmonds, R. L. 1994. Relationship between occurrence of laminated root rot and site characteristics in Douglas-fir forests of the northern Oregon Coast Range. West. J. Appl. For. 9:14-17.

Kasuga, T., White, T. J., and Taylor, J. W. 2002. Estimation of nucleotide substitution rates in Eurotiomycete fungi. Mol. Biol. Evol. 19:2318-2324.

Kotiranta, H., Mukhin, V. A., Ushakova, N., and Dai, Y.-C. 2005. Polypore (Aphyllophorales, Basidiomycetes) studies in Russia. 1. South Ural. Ann. Bot. Fenn. 42:427-451.

Łakomy, P., Broda, Z., and Werner, A. 2007. Genetic diversity of Heterobasidion spp. in Scots pine, Norway spruce and European silver fir stands. Acta Mycol. 42:203-210.

Larsen, M. J., Lombard, F. F., and Clark, J. W. 1994. Phellinus sulphurascens and the closely related $P$. weirii in North America. Mycologia 86:121-130.

Librado, P., and Rozas, J. 2009. DnaSP v5: A software for comprehensive analysis of DNA polymorphism data. Bioinformatics 25:1451-1452.

Lim, Y. W., Sturrock, R. N., Leal, I., Pellow, K. W., Yamaguchi, T., and Breuil, C. 2008. Distinguishing homokaryons and heterokaryons in Phellinus sulphurascens using pairing tests and ITS polymorphisms. Antonie Leeuwenhoek 93:99-110.

Luchi, N., Paffetti, D., Korhonen, K., Hantula, J., and Capretti, P. 2011. Genetic variation of Heterobasidion abietinum in southern Europe and the Mediterranean Basin. For. Pathol. 41:270-280.

Matheny, P. B. 2005. Improving phylogenetic inference of mushrooms with RPB1 and RPB2 nucleotide sequences (Inocybe; Agaricales). Mol. Phylogenet. Evol. 35:1-20.

Menkis, A., and Burokiene, D. 2012. Distribution and genetic diversity of the root-rot pathogen Neonectria macrodidyma in a forest nursery. For. Pathol. 42:79-83.

Mounce, I., Bier, J., and Nobles, M. K. 1940. A root-rot of Douglas fir caused by Poria weirii. Can. J. Res. 18c:522-533.

Nelson, E. E. 1971. Invasion of freshly cut Douglas-fir stumps by Poria weirii. Pacific Northwest Forest and Range Experiment Station, Portland, OR.

Ngamskulrungroj, P., Gilgado, F., Faganello, J., Litvintseva, A. P., Leal, A. L., Tsui, K. M., Mitchell, T. G., Vainstein, M. H., and Meyer, W. 2009. Genetic diversity of the Cryptococcus species complex suggests that Cryptococcus gattii deserves to have varieties. PLoS One 4:e5862.

Oliva, J., Gonthier, P., and Stenlid, J. 2011. Gene flow and inter-sterility between allopatric and sympatric populations of Heterobasidion abietinum and $H$. parviporum in Europe. For. Pathol. 41:243-252.

Parmasto, E., and Parmasto, I. 1979. The xanthochroic reaction in Aphyllophorales [Fungi, including 27 Hymenochaetaceae species, taxonomic significance]. Mycotaxon 8:201-232.

Peakall, R., and Smouse, P. E. 2006. GENALEX 6: Genetic analysis in Excel. Population genetic software for teaching and research. Mol. Ecol. Notes 6: 288-295.

Peakall, R., and Smouse, P. E. 2012. GenAlEx 6.5: Genetic analysis in Excel. Population genetic software for teaching and research-An update. Bioinformatics 28:2537-2539.

Price, M. N., Dehal, P. S., and Arkin, A. P. 2010. FastTree 2-Approximately maximum-likelihood trees for large alignments. PLoS One 5:e9490.

Reynolds, G. D. 1992. Climatic Data Summaries for the Biogeoclimatic Zones of British Columbia, Version 3. B.C. Ministry of Forests, Victoria, BC, Canada.

Riess, K., Schön, M. E., Lutz, M., Butin, H., Oberwinkler, F., and Garnica, S. 2016. On the evolutionary history of Uleiella chilensis, a smut fungus parasite of Araucaria araucana in South America: Uleiellales ord. nov. in Ustilaginomycetes. PLoS One 11:e0147107.
Ronquist, F., and Huelsenbeck, J. P. 2003. MrBayes 3: Bayesian phylogenetic inference under mixed models. Bioinformatics 19:1572-1574.

Ross-Davis, A. L., Hanna, J. W., Kim, M.-S., and Klopfenstein, N. B. 2012. Advances toward DNA-based identification and phylogeny of North American Armillaria species using elongation factor-1 alpha gene. Mycoscience 53:161-165.

Sambrook, J., Fritsch, E., and Maniatis, T. 1989. Molecular Cloning: A Laboratory Manual. Cold Spring Harbor Laboratory Press, Cold Spring Harbor, NY.

Santana, Q. C., Coetzee, M. P. A., Wingfield, B. D., Wingfield, M. J., and Steenkamp, E. T. 2016. Nursery-linked plantation outbreaks and evidence for multiple introductions of the pitch canker pathogen Fusarium circinatum into South Africa. Plant Pathol. 65:357-368.

Smith, J. M., Feil, E. J., and Smith, N. H. 2000. Population structure and evolutionary dynamics of pathogenic bacteria. BioEssays 22:1115-1122.

Smith, J. M., Smith, N. H., O’Rourke, M., and Spratt, B. G. 1993. How clonal are bacteria? Proc. Natl. Acad. Sci. USA 90:4384-4388.

Song, J., Chen, J.-J., Wang, M., Chen, Y.-Y., and Cui, B.-K. 2016. Phylogeny and biogeography of the remarkable genus Bondarzewia (Basidiomycota, Russulales). Sci. Rep. 6: Article 34568.

Song, J., and Cui, B.-K. 2017. Phylogeny, divergence time and historical biogeography of Laetiporus (Basidiomycota, Polyporales). BMC Evol. Biol. 17:102.

Stamatakis, A. 2014. RAxML version 8: A tool for phylogenetic analysis and post-analysis of large phylogenies. Bioinformatics 30:1312-1313.

Taylor, J. W., Jacobson, D. J., Kroken, S., Kasuga, T., Geiser, D. M., Hibbett, D. S., and Fisher, M. C. 2000. Phylogenetic species recognition and species concepts in fungi. Fungal Genet. Biol. 31:21-32.

Taylor, J. W., Turner, E., Townsend, J. P., Dettman, J. R., and Jacobson, D. 2006. Eukaryotic microbes, species recognition and the geographic limits of species: Examples from the kingdom Fungi. Philos. Trans. R. Soc. Lond. B Biol. Sci. 361:1947-1963.

Thies, W. G., and Sturrock, R. N. 1995. Laminated Root Rot in western North America. Gen. Tech. Rep. No. PNW-GTR-349. U.S. Department of Agriculture, Forest Service, Pacific Northwest Research Station, Portland, OR.

Thies, W. G., and Westlind, D. J. 2005. Stump removal and fertilization of five Phellinus weirii-infested stands in Washington and Oregon affect mortality and growth of planted Douglas-fir 25 years after treatment. For. Ecol. Manage. 219:242-258.

Trifinopoulos, J., Nguyen, L.-T., von Haeseler, A., and Minh, B. Q. 2016. W-IQ-TREE: A fast online phylogenetic tool for maximum likelihood analysis. Nucleic Acids Res. 44:W232-W235.

Vilgalys, R., and Hester, M. 1990. Rapid genetic identification and mapping of enzymatically amplified ribosomal DNA from several Cryptococcus species. J. Bacteriol. 172:4238-4246.

White, T. J., Bruns, T., Lee, S., and Taylor, J. 1990. Amplification and direct sequencing of fungal ribosomal RNA genes for phylogenetics. Pages 315-322 in: PCR Protocols: A Guide to Methods and Applications. M. A. Innis, D. H. Gelfand, J. J. Sninsky, and T. J. White, eds. Academic Press, Inc., San Diego, CA.

Williams, H. L., Sturrock, R. N., Islam, M. A., Hammett, C., Ekramoddoullah, A. K., and Leal, I. 2014. Gene expression profiling of candidate virulence factors in the laminated root rot pathogen Phellinus sulphurascens. BMC Genomics 15:603-618.

Zhou, L.-W., Vlasák, J., and Dai, Y.-C. 2016. Taxonomy and phylogeny of Phellinidium (Hymenochaetales, Basidiomycota): A redefinition and the segregation of Coniferiporia gen. nov. for forest pathogens. Fungal Biol. 120:988-1001. 